\title{
Bubbling the NHEK
}

\section{Pierre Heidmann}

Institut de Physique Théorique, Université Paris Saclay, CEA, CNRS, F-91191 Gif sur Yvette, France

E-mail: pierre.heidmann@ipht.fr

ABSTRACT: We build the first family of smooth bubbling microstate geometries that are asymptotic to the near-horizon region of extremal five-dimensional Kerr black holes (NHEK). These black holes arise as extremal non-supersymmetric highly-rotating D1-D5$\mathrm{P}$ solutions in type IIB string theory on $\mathrm{T}^{4} \times \mathrm{S}^{1}$. Our solutions are asymptotically NHEK in the UV and end in the IR with a smooth cap. In the context of the Kerr/CFT correspondence, these bubbling geometries are dual to pure states of the $1+1$ dimensional chiral conformal field theory dual to NHEK. Since our solutions have a bubbling structure in the IR, they correspond to an IR phase of broken conformal symmetry, and their existence supports the possibility that all the pure states whose counting gives the Kerr black hole entropy correspond to horizonless bulk configurations.

Keywords: Black Holes in String Theory, AdS-CFT Correspondence

ARXIV EPRINT: 1811.08256 


\section{Contents}

1 Introduction 1

$25 \mathrm{D}$ extremal rotating black holes in type IIB 5

2.1 The non-supersymmetric extremal D1-D5-P black holes 5

2.2 The near-horizon extremal Kerr geometry 6

$\begin{array}{lll}2.3 & \text { From } \mathrm{AdS}_{3} \times \mathrm{S}^{3} \text { to } \mathrm{WAdS}_{3} \times \mathrm{SqS}^{3} & 7\end{array}$

3 Almost-BPS bubbling geometries in type IIB $\quad 8$

3.1 Multicenter solutions in Taub-NUT 8

$\begin{array}{ll}3.2 & \text { Asymptotics of multicenter solutions } \\ 3.3 & \text { Almo }\end{array}$

$\begin{array}{lll}3.3 & \text { Almost-BPS generalized spectral flows } & 11\end{array}$

3.4 The family of almost-BPS three-supertube solutions in $\mathbb{R}^{4} \quad 12$

$\begin{array}{lll}3.4 .1 & \text { The solution } & 13\end{array}$

$\begin{array}{ll}\text { 3.4.2 The regularity conditions and conditions on the asymptotics } & 15\end{array}$

4 Asymptotically WAdS $_{3} \times \mathrm{SqS}^{3}$ bubbling geometries $\quad 17$

$\begin{array}{ll}\text { 4.1 The ultraviolet geometry } & 17\end{array}$

$\begin{array}{ll}4.2 \text { The infrared geometry } & 19\end{array}$

$\begin{array}{lll}4.3 & \text { The construction procedure } & 22\end{array}$

$\begin{array}{ll}4.4 & \text { An explicit example } \\ \end{array}$

5 Asymptotically NHEK bubbling geometries $\quad 24$

5.1 Matching the $\mathrm{WAdS}_{3}$ UV geometry to NHEK 24

$\begin{array}{ll}5.2 & \text { The construction procedure } \\ 5.3 & 25\end{array}$

$\begin{array}{lll}5.3 & \text { An explicit example } & 26\end{array}$

$\begin{array}{llr}6 & \text { Conclusions } & 27\end{array}$

$\begin{array}{ll}\text { A Derivation of } v_{I} \text { and } v_{0} & 28\end{array}$

B The explicit asymptotically WAdS $_{3} \times \mathrm{SqS}^{3}$ bubbling solution 29

C The explicit asymptotically NHEK bubbling solution 32

\section{Introduction}

The construction and analysis of smooth solitonic geometries in supergravity have attracted an intense activity over the last few years driven by the fuzzball proposal [1]. This proposal postulates that the semi-classical picture of black hole breaks down near its horizon and 
the black hole quantum state is a vector in a Hilbert space spanned by microstates approximated by smooth horizonless geometries that have the same mass, angular momentum and charges as the corresponding black hole. Most microstate geometries that have been constructed so far correspond to supersymmetric extremal black holes [2-6]. This domain of research brought many fruitful developments as the construction of the entropy enigma [7], the calculation of index-jumps when crossing walls of marginal stability [8], their application to $\mathrm{AdS}_{2}$ holography [9], the quantization of the phase space of multicenter solution [10] and many others.

From this large amount of breakthroughs, it is natural to take this line of research further towards the description of microstates of real astrophysical black holes. Several steps were taken in the construction of non-extremal non-supersymmetric black hole microstates [11-14].

In the present work, we are interested in the extreme Kerr black hole. Such a black hole in four dimensions has an angular momentum $J$ which saturates the bound $J \leq G M^{2}$ and its near-horizon geometry contains a specific warped $\mathrm{AdS}_{3}$ factor $\left(\mathrm{WAdS}_{3}\right)$, which is a particular $\mathrm{U}(1)$ fiber over $\mathrm{AdS}_{2}$.

The Kerr/CFT correspondence has been first conjectured in [15] and relates the nearhorizon geometry of extremal Kerr black hole (NHEK) to a chiral 2-dimensional conformal CFT whose the central charges are given by the angular momenta of the black hole. This conjecture correctly reproduces via Cardy's formula the Bekenstein-Hawking entropy of the black hole. Nevertheless, even if there are several possible candidates of dual "CFT 2 " as a dipole CFT [16] or as warped-CFT [17], the Kerr/CFT holographic dictionary is still poorly understood. Hence, it is very useful to have concrete examples, if not of the CFT, then of asymptotically NHEK geometries, which are bulk duals of pure CFT states.

For this purpose, it is crucial to have embeddings of NHEK in string theory as geometries arising from a system of D-branes. One of these is the six-dimensional uplift of the extremal non-supersymmetric D1-D5-P black hole in type IIB string theory on $T^{4} \times S^{1}$ [18-20]. This theory contains the extremal non-supersymmetric Kerr-Newman black hole solution with one of its angular momenta set to be zero. Its near-horizon geometry is a squashed $\mathrm{S}^{3}\left(\mathrm{SqS}^{3}\right)$ over $\mathrm{WAdS}_{3}$ which corresponds to a NHEK geometry, but the warp factor is constant, unlike for the NHEK solution in four dimensions. ${ }^{1}$

In [21], it has been shown that $\mathrm{WAdS}_{3} \times \mathrm{SqS}^{3}$ solutions, of which the NHEK spacetime is a particular example, can be obtained from $\mathrm{AdS}_{3} \times \mathrm{S}^{3}$ by a specific sequence of supergravity transformations known as $\mathcal{S} \mathcal{T U}$ transformations or generalized spectral flows [22]. This sets up the first cornerstone to build more solutions with a NHEK region in supergravity since they can be generated from more "common" six-dimensional non-supersymmetric extremal solutions in type IIB string theory (see [23] for instance).

The main goal of this paper is to apply this technique to construct smooth bubbling geometries which are asymptotically $\mathrm{WAdS}_{3} \times \mathrm{SqS}^{3}$ or more particularly asymptotically NHEK. Our methodology is to start from a family of initial non-supersymmetric extremal solutions known as almost-BPS multicenter solutions [24, 25]. As their BPS cousins, they

\footnotetext{
${ }^{1}$ In $4 \mathrm{~d}$ NHEK the warp factor depends on the polar angle.
} 
are defined by a certain number of centers in a four-dimensional Taub-NUT space which carry magnetic and electric charges corresponding to branes wrapping cycles of the transverse space. The supersymmetry is broken in a subtle way by having opposite duality between the Taub-NUT space and the fluxes [24]. Their conditions of existence being close to the BPS multicenter solutions, one can easily generate families of almost-BPS solutions using similar technique as for BPS solutions. Following the idea of [26], we will work with the family of almost-BPS solutions with three supertube centers in Taub-NUT. Each center preserves locally 16 supersymmetries. One can systematically construct such solutions and their parameter space is well-understood. Initially, these solutions are not regular in six dimensions since each species of supertube sources a different KKM dipole charge. However, as explained in [22], the three generalized spectral flows transform each of the three supertube centers to a smooth Gibbons-Hawking center. We then expect that the spectrally-flowed solutions which are the ones containing the NHEK will be smooth.

If the generalized spectral flows map one BPS solution to another, they have a much richer structure for almost-BPS solutions as the spectrally flowed solutions do not belong to the almost-BPS class any more [27]. By deriving the effect of generalized spectral flows on our three-supertube solutions, we will show in section 3 that one can indeed obtain systematically asymptotically $\mathrm{WAd}_{3} \times \mathrm{SqS}^{3}$ solutions by just constraining the initial solutions to have both angular momenta to be zero in order to be asymptotic to the specific $\mathrm{U}(1)$ fiber over $\mathrm{AdS}_{2}$ that gives the full $\mathrm{AdS}_{3}$.

Having the same metric at infinity is not the only requirement to build either asymptotically $\mathrm{WAdS}_{3} \times \mathrm{SqS}^{3}$ or asymptotically NHEK solutions. The periodicities of the angles of the squashed 3 -sphere and the angle of the warped $\mathrm{AdS}_{3}$ must have a specific form (equation (2.7) for $\mathrm{WAdS}_{3}$ with the specification (2.8) for NHEK). Imposing such periodicities to our solutions in the UV has a major impact on the smoothness of the geometry in the IR. Indeed, multicenter solutions have a $\mathbb{R}^{1,4} \times \mathrm{S}^{1}$ local geometry around each center. Thus, depending on the periodicities of the angles, conical defects can occur at these locations. A tedious smoothness analysis needs to be performed to have a smooth discrete quotient of $\mathbb{R}^{1,4} \times \mathrm{S}^{1}$ at each center.

We sketch the overall idea about our construction of smooth bubbling asymptotically $\mathrm{WAdS}_{3} \times \mathrm{SqS}^{3}$ or asymptotically NHEK geometries in figure 1 . The recipe we use to construct the solutions has the following steps:

- We start with a specific family of almost-BPS solutions. They have four centers, one is the center of Taub-NUT and the three others are two-charge supertube centers of different species. This choice of solution is just a matter of simplicity since a systematic construction is given in [26] when the solutions are supersymmetric. However, nothing prevents from taking different almost-BPS configurations. We construct asymptotically $\mathrm{AdS}_{2} \times \mathrm{S}^{1} \times \mathrm{S}^{3}$ solutions where the $\mathrm{S}^{1}$ fibration over $\mathrm{AdS}_{2}$ gives the full $\mathrm{AdS}_{3}$. Moreover, we require their left and right angular momenta to be zero.

- We perform three generalized spectral flows which transform the UV geometry to $\mathrm{WAdS}_{3} \times \mathrm{SqS}^{3}$ and preserve the bubble feature in the IR.

- We identify the periodicities at infinity of $\mathrm{WAdS}_{3} \times \mathrm{SqS}^{3}$ or NHEK and impose the absence of conical singularities at the centers. 


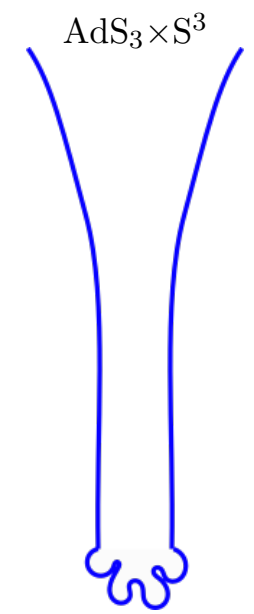

Supertube bubbles

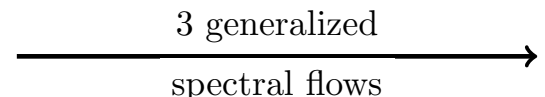

spectral flows

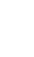




\section{$25 \mathrm{D}$ extremal rotating black holes in type IIB}

In this section we briefly review the description of non-supersymmetric three-charge overrotating Cvetic-Youm black holes which arise in the low-energy limit of type IIB string theory compactified on $T^{4} \times S^{1}$ [18-20]. We describe their near-horizon or NHEK limit and the more general family of warped-AdS $\mathrm{A}_{3}$ geometries $\left(\mathrm{WAdS}_{3}\right)$ to which those NHEK solutions belong to [16, 21, 29-31]. We also discuss the supergravity transformations which deform an $\mathrm{AdS}_{3}$ geometry to a $\mathrm{WAdS}_{3}$ geometry [21].

\subsection{The non-supersymmetric extremal D1-D5-P black holes}

We work in the context of type IIB string theory on a $T^{4} \times S^{1}$. We assume that the torus is much smaller than the one-sphere. As a consequence, the five-dimensional black hole solutions can be seen as six-dimensional black string solutions. In this paper, we consider a four-parameter family of non-supersymmetric extremal spinning black holes characterized by a mass $M$, two $\mathrm{SU}(2)_{L}$ and $\mathrm{SU}(2)_{R}$ angular momenta $J_{L}$ and $J_{R}$ and three charges $Q_{I}$ with $I=1,5, p$ as follows

$$
\begin{aligned}
M & =2 a^{2}\left(c_{1}^{2}+s_{1}^{2}+c_{5}^{2}+s_{5}^{2}+c_{p}^{2}+s_{p}^{2}\right), \\
J_{R} & =0 \\
J_{L} & =4 a^{3}\left(c_{1} c_{5} c_{p}+s_{1} s_{5} s_{p}\right), \\
Q_{I} & =4 a^{2} s_{I} c_{I}, \quad I=1,5, p,
\end{aligned}
$$

where $s_{I}=\sinh \delta_{I}$ and $c_{I}=\cosh \delta_{i}$. The Bekenstein-Hawking entropy and the left and right temperatures are

$$
\begin{aligned}
S_{B H} & =2 \pi \sqrt{J_{L}^{2}-Q_{1} Q_{5} Q_{p}}=8 \pi a^{3}\left(c_{1} c_{5} c_{p}-s_{1} s_{5} s_{p}\right), \\
T_{L} & =0 \\
T_{R} & =\frac{1}{\pi} \sqrt{1-\frac{Q_{1} Q_{5} Q_{p}}{J_{L}^{2}}} .
\end{aligned}
$$

The metric of the six-dimensional black string is [20,29]

$$
\begin{aligned}
d s_{6}^{2}= & -\left(1-\frac{4 a^{2} c_{p}^{2}}{\hat{r}^{2}+a^{2}}\right) \frac{d \hat{t}^{2}}{\sqrt{H_{1} H_{5}}}+\left(1+\frac{4 a^{2} s_{p}^{2}}{\hat{r}^{2}+a^{2}}\right) \frac{d \hat{y}^{2}}{\sqrt{H_{1} H_{5}}} \\
& +\frac{8 a^{2} s_{p} c_{p}}{\left(\hat{r}^{2}+a^{2}\right) \sqrt{H_{1} H_{5}}} d \hat{t} d \hat{y}+\left(\hat{r}^{2}+a^{2}\right) \sqrt{H_{1} H_{5}}\left(\frac{\hat{r}^{2}}{\left(\hat{r}^{2}-a^{2}\right)^{2}} d \hat{r}^{2}+d \hat{\theta}^{2}\right. \\
& \left.+\cos ^{2} \hat{\theta} d \hat{\psi}^{2}+\sin ^{2} \hat{\theta} d \hat{\phi}^{2}\right)+\frac{4 a^{4}}{\left(\hat{r}^{2}+a^{2}\right) \sqrt{H_{1} H_{5}}}\left(\cos ^{2} \hat{\theta} d \hat{\psi}+\sin ^{2} \hat{\theta} d \hat{\phi}\right)^{2} \\
& -\frac{8 a^{3}}{\left(\hat{r}^{2}+a^{2}\right) \sqrt{H_{1} H_{5}}}\left(\left(c_{1} c_{5} c_{p}+s_{1} s_{5} s_{p}\right) d t+\left(s_{1} s_{5} c_{p}+c_{1} c_{5} s_{p}\right) d \hat{y}\right)\left(\cos ^{2} \hat{\theta} d \hat{\psi}+\sin ^{2} \hat{\theta} d \hat{\phi}\right)
\end{aligned}
$$

where

$$
H_{i}=1+\frac{4 a^{2} s_{i}^{2}}{\hat{r}^{2}+a^{2}}, \quad i=1,5
$$


The coordinate $\hat{r}$ is the radial coordinate of the four-dimensional base space defined by $\hat{r}$ and the three angles $\hat{\theta}, \hat{\phi}, \hat{\psi}$ and $\hat{y}$ is the KK direction. The periodicities of the angles $\hat{y}$, $\hat{\psi}$ and $\hat{\phi}$ are $^{2}$

$$
(\hat{y}, \hat{\psi}, \hat{\phi})=\left\{\begin{array}{l}
(\hat{y}, \hat{\psi}, \hat{\phi})+2 \pi(1,0,0) \\
(\hat{y}, \hat{\psi}, \hat{\phi})+2 \pi(0,1,0) \\
(\hat{y}, \hat{\psi}, \hat{\phi})+2 \pi(0,0,1)
\end{array}\right.
$$

The geometry is asymptotically $\mathbb{R}^{1,4} \times S^{1}$ and has an event horizon at $\hat{r}_{h}=a$.

\subsection{The near-horizon extremal Kerr geometry}

The near-horizon geometry of the six-dimensional uplift of the five-dimensional black hole solutions given in (2.3) has been shown to be a near-horizon extremal Kerr geometry (NHEK) $[16,21,29,30,32]$. It is a squashed $S^{3}$ fibered over warped $\mathrm{AdS}_{3}$ with specific angle periodicities.

The near-horizon limit is obtained by changing the coordinates as follows

$$
\begin{aligned}
& t=\frac{4 \pi \epsilon}{S} \hat{t} \\
& r=\frac{\hat{r}^{2}-a^{2}}{\epsilon}, \quad y=\frac{S}{\pi Q_{1} Q_{5}}\left(\hat{y}-V_{H} \hat{t}\right), \\
& \psi=\hat{\psi}+\hat{\phi}-\frac{8 \pi a^{2}}{S} \hat{t}-\frac{4 J_{L}}{Q_{1} Q_{5}}\left(\hat{y}-V_{H} \hat{t}\right), \quad \phi=\hat{\psi}-\hat{\phi}, \quad \theta=2 \hat{\theta}
\end{aligned}
$$

where $V_{H}$ is the linear velocity $V_{H}=-\frac{8 \pi a^{3}\left(c_{1} c_{5} s_{p}-s_{1} s_{5} c_{p}\right)}{S}$. Thus, the periodicities of $y, \psi$ and $\phi$ are given by the following identifications

$$
(y, \psi, \phi)=\left\{\begin{array}{l}
(y, \psi, \phi)+2 \pi\left(T_{y},-T_{\psi}, 0\right) \\
(y, \psi, \phi)+2 \pi(0,2,0) \\
(y, \psi, \phi)+2 \pi(0,1,1)
\end{array}\right.
$$

with

$$
T_{y} \equiv \frac{S}{\pi Q_{1} Q_{5}}, \quad T_{\psi} \equiv \frac{4 J_{L}}{Q_{1} Q_{5}} .
$$

By taking the limit $\epsilon \rightarrow 0$, the near-horizon metric in terms of the above coordinates gives rise to a $\mathrm{T}^{2}$ fibration over $\mathrm{AdS}_{2} \times \mathrm{S}^{2}$

$$
\begin{aligned}
d s_{\text {NHEK }}^{2}=\frac{\kappa^{2}}{4}[ & -r^{2} d t^{2}+\frac{d r^{2}}{r^{2}}+\gamma(d y+r d t)^{2}+\gamma(d \psi+\cos \theta d \phi)^{2} \\
& \left.+2 \alpha(d y+r d t)(d \psi+\cos \theta d \phi)+d \theta^{2}+\sin ^{2} \theta d \phi^{2}\right] .
\end{aligned}
$$

where the warp constant factors $\gamma$ and $\alpha$ and the length $\kappa$ are given by

$$
\begin{aligned}
\gamma & =1+\frac{1}{\left(c_{1}^{2}+s_{1}^{2}\right)\left(c_{5}^{2}+s_{5}^{2}\right)}, \quad \alpha=\frac{1}{c_{1}^{2}+s_{1}^{2}}+\frac{1}{c_{5}^{2}+s_{5}^{2}}, \\
\kappa^{2} & =2 a^{2} \sqrt{\left(c_{1}^{2}+s_{1}^{2}\right)\left(c_{5}^{2}+s_{5}^{2}\right)} .
\end{aligned}
$$

\footnotetext{
${ }^{2}$ We have chosen the unit $R_{\hat{y}}=1$.
} 
This background belongs to the family of deformations of $\mathrm{AdS}_{3} \times \mathrm{S}^{3}$ into squashed $\mathrm{S}^{3}\left(\mathrm{SqS}^{3}\right)$ over warped $\mathrm{AdS}_{3}\left(\mathrm{WAdS}_{3}\right)$ [33-35]. However, for generic $\mathrm{WAdS}_{3} \times \mathrm{SqS}^{3}$ backgrounds, the periods $T_{y}$ and $T_{\psi}(2.7)$ are arbitrary.

One can rewrite the solutions in terms of the $\mathrm{SU}(2)_{L}$-invariant one-forms on $\mathrm{S}^{3}$

$$
\begin{aligned}
\sigma_{1} & =\cos \psi d \theta+\sin \theta \sin \psi d \phi, \quad \sigma_{2}=-\sin \psi d \theta+\sin \theta \cos \psi d \phi \\
\sigma_{3} & =d \psi+\cos \theta d \phi
\end{aligned}
$$

and the $\mathrm{SL}(2, \mathbb{R})_{L}$-invariant one forms on $\mathrm{AdS}_{3}$

$$
w_{+}=-e^{-y}\left(\frac{d r}{r}+r d t\right), \quad w_{-}=e^{y}\left(\frac{d r}{r}-r d t\right), \quad w_{3}=d y+r d t
$$

to make the $\mathrm{WAdS}_{3} \times \mathrm{SqS}^{3}$ geometry manifests

$$
d s_{N H E K}^{2}=\frac{\kappa^{2}}{4}\left(-w_{+} w_{-}+\gamma w_{3}^{2}+\sigma_{1}^{2}+\sigma_{2}^{2}+\gamma \sigma_{3}^{2}+2 \alpha w_{3} \sigma_{3}\right) .
$$

In the present paper, we want to build asymptotically $\mathrm{WAdS}_{3} \times \mathrm{SqS}^{3}$ and more particularly asymptotically NHEK bubbling geometries. For that purpose, it is interesting to review the supergravity transformations described in [21] which deform $\mathrm{AdS}_{3} \times \mathrm{S}^{3} \rightarrow \mathrm{WAdS}_{3} \times \mathrm{SqS}^{3}$ since building asymptotically $\mathrm{AdS}_{3} \times \mathrm{S}^{3}$ geometries is well-controlled and well-known.

\subsection{From $\mathrm{AdS}_{3} \times \mathrm{S}^{3}$ to $\mathrm{WAdS}_{3} \times \mathrm{SqS}^{3}$}

The sequence of supergravity transformations from an $\mathrm{AdS}_{3} \times \mathrm{S}^{3}$ spacetime to a $\mathrm{WAdS}_{3} \times \mathrm{SqS}^{3}$ has been exhaustively detailed in [21]. We just give a brief summary in this section. The transformations can be seen as a series of $\mathcal{S T U}$ transformations or equivalently as a sequence of three generalized spectral flows:

- A $\mathcal{T}$ Kähler transformation or the first generalized spectral flow.

A $\mathcal{T}$ Kähler transformation consists of a T-duality along $y$ followed by a shift of $\varphi \rightarrow \varphi+\gamma y$ where $\varphi$ can be either $\psi$ or $\phi$ and a T-duality back along $y$. We usually denote such a transformation as "TsT". The first generalized spectral flow differs from $\mathcal{T}$ transformation by a S-duality at the beginning and at the end (STsTS) and it induces the same transformation of the geometry.

- A $\mathcal{S}$ transformation or the second generalized spectral flow.

Using the previous notation, the second generalized spectral flow can be denoted as a $\mathrm{T}^{4} \mathrm{STsTST}^{4}$ transformation where the " $\mathrm{T}^{4}$ " part refers to four T-dualities on the transverse 4-torus. A $\mathcal{S}$ transformation corresponds to the same transformation with an S-duality at the beginning and at the end.

- A $\mathcal{U}$ transformation or the third generalized spectral flow.

The transformations correspond to a volume-preserving change of coordinate which simply corresponds to a shift "s" of $\varphi$. 
The two possible choices of $\varphi$ differ significantly. If the $\mathrm{AdS}_{3} \times \mathrm{S}^{3}$ background has a dual three-form field strength, the $\mathcal{S T U}$ transformations associated to this background $\left(\mathrm{SL}(2, \mathbb{R})_{L} \times \mathrm{SU}(2)_{L}\right.$ invariant) or the generalized spectral flows along $\psi$ will preserve supersymmetry and the transformed geometry will remain $\mathrm{AdS}_{3} \times \mathrm{S}^{3}$. Reversely, if the threeform field strength is anti-self-dual, the $\mathcal{S T U}$ transformations associated to this background $\left(\mathrm{SL}(2, \mathbb{R})_{L} \times \mathrm{SU}(2)_{R}\right.$ invariant) or the generalized spectral flows along $\phi$ will break supersymmetry and will transform the geometry to $\mathrm{WAdS}_{3} \times \mathrm{SqS}^{3}$ (see section 2.2 of [21] for more precision).

\section{Almost-BPS bubbling geometries in type IIB}

In the previous section, we have described the UV geometry we want to build. We have detailed how it can be obtained from an $\mathrm{AdS}_{3} \times \mathrm{S}^{3}$ UV geometry by spectral flow transformations. In the current section, we discuss our choice of non-supersymmetric extremal bubbling geometries. We work with a family of "almost-BPS" multicenter solutions in type IIB string theory on a $\mathrm{T}^{4} \times \mathrm{S}^{1}[24,25,27,36,37]$. We will be brief in the review of their general construction in section 3.1 as these solutions are discussed in great detail in [25]. In section 3.2 we explain the construction of asymptotically $\mathrm{AdS}_{3} \times \mathrm{S}^{3}$ almost-BPS solutions. Then, we review the effect of generalized spectral flows on these geometries in section 3.2 [27]. Finally, we focus on the particular family of three almost-BPS two-charge supertubes in $\mathbb{R}^{4}$ in section 3.4.

\subsection{Multicenter solutions in Taub-NUT}

The extremal almost-BPS solutions are constructed with the same ansatz of type IIB metric and matter fields as the BPS solutions:

$$
\begin{aligned}
d s_{\mathrm{ABPS}}^{2} & =-\frac{1}{Z_{p} \sqrt{Z_{1} Z_{5}}}(d t+k)^{2}+\sqrt{Z_{1} Z_{5}} d s_{4}^{2}+\frac{Z_{p}}{\sqrt{Z_{1} Z_{5}}}\left(A_{p}+d y\right)^{2}+\sqrt{\frac{Z_{1}}{Z_{5}}} d s_{T_{4}}^{2}, \\
e^{2 \Phi} & =\frac{Z_{1}}{Z_{5}} \\
B^{(2)} & =0 \\
A_{I} & =-\frac{d t+k}{Z_{I}}+a_{I}, \quad I=1,5, p,
\end{aligned}
$$

where $Z_{I}$ are the warp factors with $I=1,5, p$ encoding respectively the electric D1, D5 and $\mathrm{P}$ charge, $a_{I}$ are the magnetic one-forms, $k$ the angular momentum one-form, $A_{I}$ are the electromagnetic gauge fields and $d s_{4}$ is a hyper-Kähler four-dimensional metric which is chosen to have a Gibbons-Hawking form [38, 39]

$$
d s_{4}^{2}=V^{-1}(d \psi+A)^{2}+V\left(d r^{2}+r^{2}\left(d \theta^{2}+\sin ^{2} \theta d \phi^{2}\right)\right), \quad \star_{3} d A=d V .
$$

The Hodge star $\star_{3}$ is with respect to the three-dimensional base space, the one-form $A$ is a Kaluza-Klein gauge field and $V$ is the Taub-NUT potential:

$$
V=h_{\infty}+\frac{q}{r} \Longrightarrow A=q \cos \theta d \phi
$$


The RR three-form flux is given by

$$
F^{(3)}=d A_{1} \wedge\left(A_{p}+d y\right)-\left(\frac{Z_{5}^{5}}{Z_{1}^{3} Z_{p}^{2}}\right)^{1 / 4} \star_{5} d A_{5} .
$$

The almost-BPS equations of motion are

$$
\begin{aligned}
d a_{I} & =-\star_{4} d a_{I}, \\
d \star_{4} d Z_{I} & =\frac{\left|\epsilon_{I J K}\right|}{2} d a_{J} \wedge d a_{K}, \\
d k-\star_{4} d k & =Z_{I} d a_{I},
\end{aligned}
$$

where $\star_{4}$ is the Hodge star with respect to the Gibbons-Hawking space and $\epsilon_{I J K}$ is the Levi-Civita tensor with $\epsilon_{15 p}=1$. An almost-BPS background breaks supersymmetry by reversing the duality of the magnetic dipole field strengths $d a_{I}$ and of the angular momentum one-form $k$ (anti-self-dual) relative to the duality of the curvature of the Gibbons-Hawking space (self-dual). We expand $a_{I}$ and $k$ along the $\psi$-fiber of the Gibbons-Hawking space

$$
a_{I}=K_{I}(d \psi+A)+w_{I} \quad k=\mu(d \psi+A)+\omega .
$$

The equations of motion become equations on the three-dimensional base space

$$
\begin{aligned}
d \star_{3} d Z_{I} & =\frac{\left|\epsilon_{I J K}\right|}{2} V d \star_{3} d\left(K_{J} K_{K}\right), \\
\star_{3} d w_{I} & =V d K_{I}-K_{I} d V, \\
\star_{3} d \omega & =V Z_{I} d K_{I}-d(\mu V), \\
d \star_{3} d(\mu V) & =-d\left(V Z_{I}\right) \star_{3} d K_{I}
\end{aligned}
$$

The solutions are determined by eight harmonic functions $\left\{V, K_{1}, K_{5}, K_{p}, L^{1}, L^{5}, L^{p}, M\right\}$ where the functions $L_{I}$ source the warp factors $Z_{I}$ and $M$ sources $\mu$. Each harmonic function is sourced by $n+1$ centers on the three-dimensional base space. In the present paper, we are interested in axisymmetric configurations where the centers are denoted by a coordinate $a_{i}$ on the $z$ axis in $\mathbb{R}^{3}, i=0 \ldots n$ with $a_{0}=0$,. The harmonic functions carry a charge at each center. We use the following notation:

$$
K^{I}=k_{\infty}^{I}+\sum_{i=0}^{n} \frac{k_{i}^{I}}{r_{i}}, \quad L_{I}=l_{\infty}^{I}+\sum_{i=0}^{n} \frac{Q_{i}^{(I)}}{r_{i}}, \quad M=m_{\infty}+\sum_{i=0}^{n} \frac{m_{i}}{r_{i}},
$$

where $r_{i}$ is the three-dimensional distance to the $i^{\text {th }}$ center $r_{i}=\sqrt{r^{2}+a_{i}^{2}-2 r a_{i} \cos \theta}$. From those expressions and the equations (3.7), one can derive the general form of the warp factors [25]

$$
Z_{I}=L_{I}+\frac{\left|\epsilon_{I J K}\right|}{2} \sum_{j, k}\left(h_{\infty}+\frac{q r}{a_{j} a_{k}}\right) \frac{k_{j}^{J} k_{k}^{K}}{r_{j} r_{k}},
$$

and the generic expression of the angular momentum one-form $k$ given by $\omega$ and $\mu$ can be found in [25]. 
In anticipation of the computation of generalized spectral flows in section 3.3, we define four magnetic and electric one-forms, $v_{I}$ and $v_{0}$, determined by the following equations

$$
\begin{aligned}
& \star_{3} d v_{I} \equiv-d Z_{I}+\frac{\left|\epsilon_{I J K}\right|}{2}\left(V d\left(K_{J} K_{K}\right)-K_{J} K_{K} d V\right), \\
& \star_{3} d v_{0} \equiv K_{I} d Z_{I}-Z_{I} d K_{I}+K_{1} K_{5} K_{p} d V-V d\left(K_{1} K_{5} K_{p}\right) .
\end{aligned}
$$

These solutions do not necessarily correspond to physical geometries. For that purpose, regularity conditions have to be satisfied [25]:

- Since the angular momentum one-form $\omega$ is proportional to $d \phi, \omega$ must vanish on the z-axis where $\phi$ degenerates to avoid Dirac-Misner string singularities. This imposes $n+1$ bubble equations on the distances between the centers (see [25] for the generic equations).

- The absence of closed timelike curves requires the positivity of the quartic invariant:

$$
\mathcal{I}_{4} \equiv Z_{1} Z_{5} Z_{p} V-\mu^{2} V^{2}>0
$$

Those regularity conditions constrain significantly the parameter space of the solutions. Once they are satisfied, we have a family of extremal non-supersymmetric solutions in six dimensions which cap off in the IR. The solutions are regular everywhere but they might have singularities at the centers. These corresponding regularity conditions depend on the nature of the centers and will be discussed for supertube centers in section 3.4.2.

\subsection{Asymptotics of multicenter solutions}

In section 2.3 we have described a procedure to go from an $\mathrm{AdS}_{3} \times \mathrm{S}^{3} \mathrm{UV}$ region to $\mathrm{WAdS}_{3} \times \mathrm{SqS}^{3}$. We discuss now the asymptotics of a bubbling almost-BPS solution. We derive the conditions to be asymptotic to the specific $\mathrm{S}^{1}$ fibration over $\mathrm{AdS}_{2} \times \mathrm{S}^{3}$ that gives the full $\mathrm{AdS}_{3} \times \mathrm{S}^{3}$ :

$$
d s_{\infty}^{2} \propto-r^{2} d t^{2}+\frac{d r^{2}}{r^{2}}+(d y+r d t)^{2}+d \Omega_{3}^{2}
$$

The asymptotics of a multicenter solution is given by the large-distance behavior of the warp factors $Z_{I}$, the Gibbons-Hawking function $V$ and the angular momentum one-form $k$. We already assume that the constant term in $V$ is zero which is a straightforward necessary condition to have an AdS factor at infinity. The series expansion of $Z_{I}, V$ and $k$ involves the constant terms $l_{\infty}^{I}$ and $m_{\infty}$, the D1, D5 and P charges and the left and right angular momenta of the solution which we denote as $q_{1}, q_{5}, q_{p}, j_{L}$ and $j_{R}$ :

$$
\begin{aligned}
Z_{1} \underset{r \rightarrow \infty}{\sim} l_{\infty}^{1}+\frac{q_{1}}{r}, \quad Z_{5} \underset{r \rightarrow \infty}{\sim} l_{\infty}^{5}+\frac{q_{5}}{r}, \quad Z_{p} \underset{r \rightarrow \infty}{\sim} l_{\infty}^{p}+\frac{q_{p}}{r}, \\
k \underset{r \rightarrow \infty}{\sim} \frac{j_{R}+j_{L} \cos \theta}{r} d \psi+q \frac{j_{L}+j_{R} \cos \theta}{r} d \phi, \quad V \underset{r \rightarrow \infty}{\sim} \frac{q}{r} .
\end{aligned}
$$

From the metric (3.1), we see that an asymptotic behavior as (3.12) can be achieved by imposing that all the constant terms and the left and right angular momenta are strictly zero. ${ }^{3}$

\footnotetext{
${ }^{3}$ Having no angular momentum essentially means that $\mu$ and $\omega$ decay as $r^{-2}$.
} 
The requirement $h_{\infty}=0$ makes the Taub-NUT space to be a trivial $\mathbb{R}^{4}$. The supersymmetry breaking obtained by an opposite direction between the base space and the gauge fields does not hold anymore and the solution can be mapped to a BPS solution by interchanging $\phi \leftrightarrow \psi[21,24,36]$. However, as explained in section 2.3, one can still count on the generalized spectral flows to break supersymmetry.

Furthermore, in our language, having no constant terms in the harmonic functions makes the solution to be asymptotically $\mathrm{AdS}_{2} \times \mathrm{S}^{1}$ rather than $\mathrm{AdS}_{3}$ [9]. This is just a matter of convention since one can consider the $\mathrm{AdS}_{3} \times \mathrm{S}^{3}$ metric (3.12) as a U(1) fiber on an $\mathrm{AdS}_{2} \times \mathrm{S}^{3}$.

Once the conditions on the constant terms and the angular momenta are satisfied, one can perform a sequence of three generalized spectral flows. They will transform the IR geometry by keeping it bubbling and by transforming the supertube center to smooth center [22]. They will transform the UV geometry from (3.12) to a $\mathrm{WAdS}_{3} \times \mathrm{SqS}^{3}$ geometry $(2.9)$.

\subsection{Almost-BPS generalized spectral flows}

The three generalized spectral flows detailed in 2.3 can be translated in the formalism of multicenter solutions as transformations of the NSNS and RR fields. As explained in the previous section, our choice of constant terms makes our solutions to be BPS by interchanging $\phi \leftrightarrow \psi$. Consequently, the generalized spectral flows corresponding to a shift $\phi \rightarrow \phi+\gamma y$ produce the usual BPS generalized spectral flows which consist in interchanging linearly the harmonic functions and which will preserve the supersymmetry [40-42]. However, the generalized spectral flows corresponding to a shift $\psi \rightarrow \psi+\gamma y$ produce the expected supersymmetry breaking and will allow to go from the almost-BPS class of solutions to different non-supersymmetric classes [27, 43] as the family of asymptotically $\mathrm{WAdS}_{3}$ solutions. We now review the transformation rules of generalized spectral flows on the NSNS and RR fields [21, 27]. Let us define the three constant shifts $\gamma_{1}, \gamma_{5}$ and $\gamma_{p}$ of the three types of spectral flows and the following new functions

$$
T_{I} \equiv 1+\gamma_{I} K_{I}, \quad N_{I}=\frac{\left|\epsilon_{I J K}\right|}{2} \gamma_{I}^{2} Z_{J} Z_{K}+V T_{I}^{2} Z_{I}-2 \gamma_{I} V T_{I} \mu, \quad I=1,5, p,
$$

and we define the usual short-hand notations $K^{3}=K_{1} K_{5} K_{p}, T^{3}=T_{y} T_{5} T_{p}, N^{3}=N_{1} N_{5} N_{p}$, $\gamma^{3}=\gamma_{1} \gamma_{5} \gamma_{p}$ and $Z^{3}=Z_{1} Z_{5} Z_{p}$. The spectrally flowed 6-dimensional metric and the matter gauge fields are given by [27]

$$
\begin{aligned}
d \widetilde{s}_{6 d}^{2}= & -\frac{1}{\widetilde{Z}_{p} \sqrt{\widetilde{Z}_{1} \widetilde{Z}_{5}}}(d t+\widetilde{\mu}(d \psi+\widetilde{A})+\omega)^{2}+\frac{\widetilde{Z}_{p}}{\sqrt{\widetilde{Z}_{1} \widetilde{Z}_{5}}}\left(\widetilde{A}_{p}+d y\right)^{2} \\
& +\sqrt{\widetilde{Z}_{1} \widetilde{Z}_{5}}\left(\widetilde{V}^{-1}(d \psi+\widetilde{A})^{2}+\widetilde{V} d s\left(\mathbb{R}^{3}\right)^{2}\right) \\
\widetilde{A}_{I}= & -\frac{d t+\omega}{\widetilde{W}_{I}}+\widetilde{P}_{I}(d \psi+\widetilde{A})+\widetilde{w}_{I}
\end{aligned}
$$


where

$$
\begin{aligned}
\widetilde{V}= & {\left[T^{6} V^{2}+8 \gamma^{3} T^{3} V \mu-T^{3} V\left(\left|\epsilon_{I J K}\right| \gamma_{J} \gamma_{K} T_{I} Z_{I}\right)\right.} \\
& \left.+\frac{\left|\epsilon_{I J K}\right|}{2} \gamma_{J}^{2} \gamma_{K}^{2} T_{I}^{2} Z_{I}^{2}-\left|\epsilon_{I J K}\right| \gamma_{I}^{2} \gamma_{J} \gamma_{K} T_{J} Z_{J} T_{K} Z_{K}\right]^{1 / 2}, \\
\widetilde{A}= & A-\gamma_{I} w_{I}-\frac{\left|\epsilon_{I J K}\right|}{2} \gamma_{J} \gamma_{K} v_{I}+\gamma^{3} v_{0}, \\
\widetilde{Z}_{I}= & \frac{N_{I}}{\widetilde{V}}, \\
\widetilde{\mu}= & \widetilde{V}^{-2}\left(T^{3} V^{2} \mu-\gamma^{3} Z^{3}+\frac{\left|\epsilon_{I J K}\right|}{2} \gamma_{J} \gamma_{K} Z_{I} T_{I} V \mu-\frac{\left|\epsilon_{I J K}\right|}{2} \gamma_{I} V T_{J} Z_{J} T_{K} Z_{K}\right), \\
\widetilde{W}_{I}= & \frac{N_{I}}{T^{3} V+\frac{\left|\epsilon_{I J K}\right|}{2} \gamma_{J} \gamma_{K} T_{I} Z_{I}-\left|\epsilon_{I J K}\right| \gamma_{I} \gamma_{J} T_{K} Z_{K}}, \\
\widetilde{P}_{I}= & \frac{V Z_{I} T_{I} K_{I}+\frac{\left|\epsilon_{I J K}\right|}{2} \gamma_{I} Z_{J} Z_{K}-\left(2 T_{I}-1\right) V \mu}{N_{I}}, \\
\widetilde{w}_{I}= & w_{I}+\left|\epsilon_{I J K}\right| \gamma_{J} v_{K}-\frac{\left|\epsilon_{I J K}\right|}{2} \gamma_{J} \gamma_{K} v_{0} .
\end{aligned}
$$

Generalized spectral flows produce a non-trivial modification of the functions. However, the spectrally flowed extremal solutions still satisfy the regularity conditions. Indeed, the one-form $\omega$ is unchanged which guarantees the absence of Dirac-Misner string at $\theta=0, \pi$. Moreover, the quartic invariant is preserved under spectral flows $\tilde{\mathcal{I}}_{4}=\mathcal{I}_{4}$. Hence, a regular almost-BPS multicenter solution is transformed by a generic spectral flows to a regular extremal non-supersymmetric solution.

Furthermore, if the initial almost-BPS solution has curvature singularities at the centers which happens for supertube centers of type $I=1,5$, the corresponding generalized spectral flows will transform the singular local geometries to quotients of $\mathbb{R}^{4} \times \mathrm{S}^{1}$ [22].

Nevertheless, conical singularities related to the angle periodicities can still occur at these locations. Indeed, the NHEK angle periodicities or the $\mathrm{WAdS}_{3}$ angle periodicities (2.7) imposed in the UV can spoil the periodicities at the centers where the three-sphere shrinks and conical singularities can emerge. Those are the only regularity conditions we need to worry about after spectral flows.

We now have all the basic ingredients to construct an extremal non-supersymmetric geometry which caps off smoothly in the IR and is asymptotically NHEK or WAdS 3 . Working with the most generic almost-BPS multicenter solutions can lead to very complicated regularity conditions that are hard to analyze. That is why, we will focus our work on the family of four-center solutions of three two-charge supertubes in $\mathbb{R}^{4}$. As explain in [26], a systematic construction can be performed for BPS configurations. We extend the construction in the almost-BPS context in the next section.

\subsection{The family of almost-BPS three-supertube solutions in $\mathbb{R}^{4}$}

We consider the family of almost-BPS solutions with three two-charge supertubes and a $\mathbb{R}^{4}$ base space. For the BPS solutions, a systematic construction of the family have been 
performed [26]. As explained in section 3.2, almost-BPS solutions in $\mathbb{R}^{4}$ do not literally break supersymmetry as they can be mapped to BPS solutions [24, 36]. Those solutions are BPS, but can be constructed either as BPS or as almost-BPS solutions. Since we need the second for the spectral flows to break supersymmetry, we will do it here.

Thus, the extension of the construction is just a matter of rewriting carefully in the context of almost-BPS solutions. In this section, we apply the general results obtained in section 3.1 to our specific family of solutions. We will consider an axisymmetric supertube configuration. We first detail how the NSNS and RR fields are sourced by such a configuration. We then derive the regularity conditions and show that they can be systematically satisfied.

\subsubsection{The solution}

A type " $I$ " supertube, with $I=1,5, p$, has a singular magnetic source in $K^{I}$, two singular electric sources in $Z_{J}$ and $Z_{K}$ with $I \neq J \neq K$ and one angular-momentum charge in $M$ [44]. The six-dimensional metric and the matter fields are still given by (3.1). We assume that the $\mathbb{R}^{4}$ center is at the origin of the space and that a supertube of type 1 is at a second center with coordinate $a_{1}$ on the z-axis, a supertube of type 5 is at a third center with coordinate $a_{5}$ and a supertube of type $\mathrm{p}$ is at a fourth center with coordinate $a_{p}$. We consider that $a_{I}>0$. We denote by $r_{I}$ the three-dimensional distance from the $I^{\text {th }}$ center $r_{I} \equiv \sqrt{r^{2}+a_{I}^{2}-2 r a_{I} \cos \theta}$. We use the following notation for the eight harmonic functions ${ }^{4}$

$$
\begin{aligned}
V & =\frac{q}{r}, & M & =m_{\infty}+\frac{m_{0}}{r}+\frac{m_{1}}{r_{1}}+\frac{m_{5}}{r_{5}}+\frac{m_{p}}{r_{p}}, \\
K^{1} & =k_{\infty}^{1}+\frac{a_{1} \kappa_{1}}{q r_{1}}, & L_{1} & =\frac{Q_{5}^{(1)}}{r_{5}}+\frac{Q_{p}^{(1)}}{r_{p}}, \\
K^{5} & =k_{\infty}^{5}+\frac{a_{5} \kappa_{5}}{q r_{5}}, & L_{5} & =\frac{Q_{1}^{(5)}}{r_{1}}+\frac{Q_{p}^{(5)}}{r_{p}}, \\
K^{p} & =k_{\infty}^{p}+\frac{a_{p} \kappa_{p}}{q r_{p}}, & L_{p} & =\frac{Q_{1}^{(p)}}{r_{1}}+\frac{Q_{5}^{(p)}}{r_{5}},
\end{aligned}
$$

We have defined on purpose the "effective" dipole charges $\kappa_{I}$ as a function of the charges in $K^{I}: \quad \kappa_{I}=\frac{q k_{I}}{a_{I}}$. Those effective dipole charges have been argued to be the local magnetic charges obtained by integrating the magnetic dipole strength $d a_{I}$ around the center $[25,37]$. Using the expression of the warp factors (3.9) and the general expressions

\footnotetext{
${ }^{4}$ We remind that all the constant terms in $V$ and $L^{I}$ have been set to zero to have an asymptotically $\mathrm{AdS}_{2} \times \mathrm{S}^{1} \times \mathrm{S}^{3}$ solution.
} 
for $\mu$ and $\omega$ in the [25], we obtain for our specific solutions

$$
\begin{aligned}
Z_{1}= & \frac{Q_{5}^{(1)}}{r_{5}}+\frac{Q_{p}^{(1)}}{r_{p}}+\frac{\kappa_{5} \kappa_{p}}{q} \frac{r}{r_{5} r_{p}}, \\
Z_{5}= & \frac{Q_{1}^{(5)}}{r_{1}}+\frac{Q_{p}^{(5)}}{r_{p}}+\frac{\kappa_{1} \kappa_{p}}{q} \frac{r}{r_{1} r_{p}}, \\
Z_{p}= & \frac{Q_{1}^{(p)}}{r_{1}}+\frac{Q_{5}^{(p)}}{r_{5}}+\frac{\kappa_{1} \kappa_{5}}{q} \frac{r}{r_{1} r_{5}}, \\
\mu= & \sum_{I} \sum_{J \neq I} \frac{Q_{J}^{(I)} \kappa_{I}}{2 q} \frac{r^{2}+a_{I} a_{J}-2 a_{I} r \cos \theta}{\left(a_{J}-a_{I}\right) r_{I} r_{J}}+\frac{\kappa_{1} \kappa_{5} \kappa_{p}}{q^{2}} \frac{r^{2} \cos \theta}{r_{1} r_{5} r_{p}}+\frac{r M}{q}, \\
\omega= & {\left[\sum_{I} \sum_{J \neq I} \frac{Q_{J}^{(I)} \kappa_{I}}{2} \frac{r\left(a_{J}+a_{I} \cos 2 \theta\right)-\left(r^{2}+a_{I} a_{J}\right) \cos \theta}{\left(a_{J}-a_{I}\right) r_{I} r_{J}}+\frac{\kappa_{1} \kappa_{5} \kappa_{p}}{q} \frac{r^{2} \sin ^{2} \theta}{r_{1} r_{5} r_{p}}\right.} \\
& \left.+\omega_{0}-\sum_{I} m_{I} \cos \theta_{I}-m_{0} \cos \theta\right] d \phi,
\end{aligned}
$$

where we have defined the polar angles $\theta_{I}$ which correspond to the angle with the z-axis with respect to the center $I$

$$
\cos \theta_{I} \equiv \frac{r \cos \theta-a_{I}}{r_{I}}
$$

In order to analyze the spectrally-flowed solutions, we need to compute the electromagnetic gauge fields $A_{I}$ of the initial solutions

$$
\begin{aligned}
& w_{I}=\left(\kappa_{I} \frac{r-a_{I} \cos \theta}{r_{I}}-q k_{\infty}^{I} \cos \theta\right) d \phi, \\
& A_{I}=-\frac{d t+\mu(d \psi+q \cos \theta d \phi)+\omega}{Z_{I}}+K_{I}(d \psi+q \cos \theta d \phi)+w_{I} .
\end{aligned}
$$

For the same reason, the electromagnetic one-forms $v_{0}$ and $v_{I}$ involved in the spectral flow transformations of the gauge fields must be derived. This has not been done yet in the literature. We solve their equations (3.10) in the context of our solutions in the appendix A: ${ }^{5}$

$$
\begin{gathered}
v_{I}=\frac{\left|\epsilon_{I J K}\right|}{2}\left[-q k_{\infty}^{J} k_{\infty}^{K} \cos \theta+2 k_{\infty}^{J} \kappa^{K} t_{J}^{(1)}-2 Q_{J}^{(I)} t_{J}^{(2)}-\frac{\kappa_{J} \kappa_{K}}{q} t_{J K}^{(3)}\right] d \phi, \\
v_{0}=\frac{\left|\epsilon_{I J K}\right|}{6}\left[q k_{\infty}^{I} k_{\infty}^{J} k_{\infty}^{K} \cos \theta+6 k_{\infty}^{I} Q_{J}^{(I)} t_{J}^{(1)}-3 k_{\infty}^{I} k_{\infty}^{J} \kappa_{K} t_{K}^{(2)}\right. \\
\left.\quad+6 \frac{\kappa_{I} Q_{J}^{(I)}}{q} t_{I J}^{(3)}+3 \frac{k_{\infty}^{I} \kappa_{J} \kappa_{K}}{q} t_{J K}^{(4)}+\frac{\kappa_{I} \kappa_{J} \kappa_{K}}{q^{2}} t_{I J K}^{(5)}\right] d \phi
\end{gathered}
$$

\footnotetext{
${ }^{5}$ This result can be easily generalized to generic multicenter almost-BPS solutions.
} 
with

$$
\begin{array}{rlrl}
t_{I}^{(1)} & \equiv \cos \theta_{I}, & t_{I J}^{(3)} \equiv \frac{a_{I}}{a_{J}-a_{I}} \frac{r^{2}+a_{I} a_{J}-\left(a_{I}+a_{J}\right) r \cos \theta}{r_{I} r_{J}} \\
t_{I}^{(2)} & \equiv \frac{r-a_{I} \cos \theta}{r_{I}}, \quad t_{I J}^{(4)} \equiv \frac{\left(r^{2}+a_{I} a_{J}\right) \cos \theta-\left(a_{I}+a_{J}\right) r}{r_{I} r_{J}}, \\
t_{I J K}^{(5)} & \equiv \frac{r^{3}+r\left(a_{I} a_{J}+a_{I} a_{K}+a_{J} a_{K}\right)-\left(r^{2}\left(a_{I}+a_{J}+a_{K}\right)+a_{I} a_{J} a_{K}\right) \cos \theta}{r_{I} r_{J} r_{K}} .
\end{array}
$$

At this point, we have the full description of the almost-BPS solutions we will use as input for our construction. In general, most of the solutions in this class are not regular. We investigate the regularity conditions in the next section.

\subsubsection{The regularity conditions and conditions on the asymptotics}

- 16-supercharge regular two-charge supertube:

A single two-charge supertube with dipole charge corresponding to, say, $K_{p}$ gives a regular six-dimensional metric if its angular-momentum charge is fixed to be $m_{p}=\frac{q Q_{p}^{(1)} Q_{p}^{(5)}}{2 a_{p} \kappa_{p}}[25$, 45]. Imposing such a condition to the three types of supertubes, we obtain the usual supertube regularity

$$
m_{I}=\frac{\left|\epsilon_{I J K}\right|}{2} \frac{q Q_{I}^{(J)} Q_{I}^{(K)}}{2 a_{I} \kappa_{I}}, \quad I=1,5, p
$$

- Absence of Dirac-Misner strings at the centers:

The absence of Dirac-Misner string singularities in $\omega$ requires $\left.\omega\right|_{\theta=0, \pi}=0$. From (3.18), this gives one condition on the constant term in $\omega$ and four bubble equations

$$
\begin{aligned}
\omega_{0} & =0 \\
2 m_{0} & =\frac{\Gamma_{15}}{a_{1}-a_{5}}+\frac{\Gamma_{1 p}}{a_{1}-a_{p}}+\frac{\Gamma_{5 p}}{a_{5}-a_{p}}, \\
\frac{q Q_{1}^{(5)} Q_{1}^{(p)}}{a_{1} \kappa_{1}} & =\frac{\Gamma_{15}}{\left|a_{1}-a_{5}\right|}+\frac{\Gamma_{1 p}}{\left|a_{1}-a_{p}\right|}, \\
\frac{q Q_{5}^{(1)} Q_{5}^{(p)}}{a_{5} \kappa_{5}} & =\frac{\Gamma_{51}}{\left|a_{1}-a_{5}\right|}+\frac{\Gamma_{5 p}}{\left|a_{5}-a_{p}\right|}, \\
\frac{q Q_{p}^{(1)} Q_{p}^{(5)}}{a_{p} \kappa_{p}} & =\frac{\Gamma_{p 1}}{\left|a_{1}-a_{p}\right|}+\frac{\Gamma_{p 5}}{\left|a_{5}-a_{p}\right|},
\end{aligned}
$$

where $\Gamma_{I J} \equiv \kappa_{I} Q_{J}^{(I)}-\kappa_{J} Q_{I}^{(J)}$.

- Absence of closed timelike curves:

The absence of closed timelike curves in the rest of the space requires the positivity of the quartic invariant $\mathcal{I}_{4}$ (3.11). This condition is in general very complicated to check directly since it is not an algebraic condition. However, it has been showed in [26] that it can be 
systematically satisfied for our configurations. We just review briefly here the mechanism. A necessary condition for $\mathcal{I}_{4}>0$ is to have

$$
Z_{I} V>0, \quad I=1,5, p, \quad \mu \underset{r \rightarrow \infty}{\rightarrow} 0 .
$$

Imposing $\mu \rightarrow 0$ requires straightforwardly that $m_{\infty}=0$. Furthermore, if we expand $Z_{I} V$ around the poles which is sufficient to prove (3.25) we get the following conditions:

$$
q \frac{Q_{J}^{(I)}}{a_{J}}+\frac{\kappa_{I} \kappa_{J}}{\left|a_{I}-a_{J}\right|}>0 \quad \text { and } \quad q\left(\frac{Q_{J}^{(I)}}{a_{J}}+\frac{Q_{K}^{(I)}}{a_{K}}\right)>0, \quad I \neq J \neq K .
$$

This is trivially solved by taking all the supertube charges and $q$ to be positive. However, if one sums the three last bubble equations (3.24), at least one supertube dipole charge needs to be negative. Let us consider only one negative charge, say $\kappa_{5}$. The conditions (3.26) will just define a significantly large domain of possible values.

A priori, the condition (3.25) only guarantees that $Z_{1} Z_{5} Z_{p} V>0$ which does not necessarily mean $\mathcal{I}_{4}>0$. However, from the construction of $\mu(3.7)$, this is practically always sufficient. Moreover, we want our initial solutions to satisfy $j_{L}=j_{R}=0$ as explained in section 3.2. So $\mu$ decays as $r^{-2}$ rather than $r^{-1}$ which gives a stronger evidence for this fact.

- Conditions on the asymptotics

Our solutions need to be asymptotic to the specific $S^{1}$ fibration over $\mathrm{AdS}_{2}$ giving $\mathrm{AdS}_{3}$ (3.12). For that purpose, the constant terms in the warp factors $Z_{I}$ and in the Gibbons-Hawking function $V$ have been set to zero at the beginning (3.18). Furthermore, to obtain the specific $\mathrm{S}^{1}$ fibration, the right and left angular momenta must be zero as detailed in section 3.2. They can be derived from the asymptotic behaviour of $\mu$

$$
\mu \underset{r \rightarrow \infty}{\sim} \frac{j_{R}+j_{L} \cos \theta}{r} .
$$

The $\mathrm{AdS}_{2}$ throat has an infinite length due to the vanishing constant terms. This means that $j_{R}=0$ is straightforwardly satisfied. We obtain $j_{L}$ from (3.18):

$$
j_{L}=\frac{2}{q}\left(\frac{\kappa_{1} \kappa_{5} \kappa_{p}}{q}+\sum_{I \neq J \neq K} \frac{Q_{I}^{(J)} Q_{I}^{(K)}}{\kappa_{I}}+\frac{1}{2} \sum_{I \neq J} \kappa_{I} Q_{J}^{(I)}\right)=0 .
$$

Our initial almost-BPS solutions must satisfy this equation before applying the sequence of generalized spectral flows.

In this section, we have described in full detail the family of extremal nonsupersymmetric three-supertube solutions with a flat $\mathbb{R}^{4}$ base space and with zero left and right angular momenta. We have shown a procedure to construct systematically bubbling solutions of this type. We expect from section 2.3 that acting with three generalized spectral flows on those initial solutions will produce our expected smooth bubbling asymptotically $\mathrm{WAdS}^{3} \times \mathrm{SqS}^{3}$ or NHEK geometries. We will discuss this construction in the next section. 


\section{Asymptotically $\mathrm{WAdS}_{3} \times \mathrm{Sq}^{3}$ bubbling geometries}

We start with the solutions constructed in the previous section. We will perform three generalized spectral flows parametrized by the constant shifts $\gamma_{1}, \gamma_{5}$ and $\gamma_{p}$. Even if the transition from an $\mathrm{AdS}_{3}$ to a $\mathrm{WAdS}_{3}$ with generalized spectral flows seems to be straightforward from the point of view of section 2.3, things get more complicated for a bubbling geometry and we will need to massage the initial solutions and the spectral flows to satisfy different regularity conditions in the UV and IR geometries:

- The spectrally flowed UV geometry differs from a $\mathrm{WAdS}_{3} \times \mathrm{SqS}^{3}$ geometry by the angle periodicities (2.7) even if we start with an initial solution which is asymptotic to the right $\mathrm{S}^{1}$ fibration over $\mathrm{AdS}_{2}$. In section 4.1, we will deal with the spectral flow parameters and the parameters of the initial solution to get a UV geometry exactly identified as a $\mathrm{WAdS}_{3} \times \mathrm{SqS}^{3}$ geometry with the right angle periodicities (2.7).

- The spectrally flowed IR geometry is a smooth bubbling geometry. However, the modification of the angle periodicities in the UV region changes drastically the periods around the centers. Conical singularities can occur at the centers where the $\mathrm{S}^{3}$ shrinks. We will show in section 4.2 that one can still systematically build geometries where the UV angle periods do not yield to conical singularities.

Several attempts on building bubbling geometries with a NHEK or $\mathrm{WAdS}_{3} \times \mathrm{SqS}^{3}$ region have been performed in the previous work [21, 23]. In [21], only very specific $\mathrm{WAdS}_{3}$ geometries with limited field contents have been built. Furthermore, in both papers, the NHEK regions were built in the deep IR and the issue of conical singularities which can occur at the centers was not tackled. Here we give all the details of the construction of the largest known family of smooth general solutions with a $\mathrm{WAdS}_{3} \times \mathrm{SqS}^{3} \mathrm{UV}$.

\subsection{The ultraviolet geometry}

We start with a solution of the family of almost-BPS solutions (detailed in 3.4) with all the constraints and regularity conditions satisfied. Thus, the asymptotic behavior of the initial solution is

$$
Z_{I} \sim \frac{q_{I}}{r}, \quad K^{I} \sim k_{\infty}^{I}, \quad V \sim \frac{q}{r}, \quad \mu=\omega=\mathcal{O}\left(r^{-2}\right), \quad r \gg 1,
$$

By applying the spectral flow transformation rules (3.16), the solution after three generalized spectral flows has the following asymptotic expansion:

$$
\begin{aligned}
\widetilde{Z}_{I} & \sim \frac{\widetilde{q}_{I}}{r}, \quad \widetilde{V} \sim \frac{\widetilde{q}}{r}, & \widetilde{\mu} \sim \frac{\widetilde{J}}{r}, \quad \widetilde{W}_{I} \sim \frac{\widetilde{\chi}_{I}}{r}, & \widetilde{P}_{I} \sim \widetilde{k}_{\infty}^{I} \\
\widetilde{A} & \sim\left(\widetilde{A}_{\infty}^{(0)}+\widetilde{A}_{\infty} \cos \theta\right) d \phi, & \widetilde{w}_{I} \sim\left(\widetilde{w}_{I \infty}^{(0)}+\widetilde{w}_{I \infty} \cos \theta\right) d \phi, & r \rightarrow \infty,
\end{aligned}
$$

where each tilded quantity in the right-hand side is a constant which can be derived from (3.16) as a function of the asymptotic values of the initial solution (4.1). Since these functions are rather complicated and of minor interest, we did not write them down 
in their general forms. However, it is noteworthy that $\widetilde{q}$ is generically a square root of a polynomial function. In anticipation of the constraints demanded by the regularity around the centers, one needs to impose all the quantities to be at least rational. For that purpose, we fix the polynomial to be a perfect square. Two simple choices are: $\gamma_{p}=0$ and $\gamma_{p}=-\frac{1}{k_{\infty}^{p}}$. We have analyzed both possibilities and it happens that the second one leads to simpler solutions. From now on, we suppose that $\gamma_{p}=-\frac{1}{k_{\infty}^{p}}$. We define the constants

$$
t_{\infty}^{I} \equiv 1+k_{\infty}^{I} \gamma_{I}
$$

Then, we have ${ }^{6}$

$$
\begin{array}{rlrl}
\widetilde{q} & =\left|\frac{q_{1} \gamma_{5} t_{\infty}^{1}-q_{5} \gamma_{1} t_{\infty}^{5}}{k_{\infty}^{p}}\right|, & \widetilde{q}_{I} & =\frac{q q_{I}\left(t_{\infty}^{I}\right)^{2}+\frac{\left|\epsilon_{I J K}\right|}{2} \gamma_{I}^{2} q_{J} q_{K}}{\widetilde{q}}, \\
\widetilde{J} & =\frac{q_{1} q_{5}}{k_{\infty}^{p} \widetilde{q}^{2}}\left(\gamma_{1} \gamma_{5} q_{p}+q t_{\infty}^{1} t_{\infty}^{5}\right), & \widetilde{\chi}_{I}=\left|\epsilon_{I J K}\right| \frac{2 \widetilde{q} \widetilde{q}_{I}}{\gamma_{J} \gamma_{K} t_{\infty}^{I} q_{I}-2 \gamma_{I} \gamma_{J} t_{\infty}^{K} q_{K}}, \\
\widetilde{k}_{\infty}^{I} & =\frac{q q_{I} k_{\infty}^{I} t_{\infty}^{I}+\frac{\left|\epsilon_{I J K}\right|}{2} \gamma_{I} q_{J} q_{K}}{q q_{I}\left(t_{\infty}^{1}\right)^{2}+\frac{\left|\epsilon_{I J K}\right|}{2} \gamma_{I}^{2} q_{J} q_{K}}+\frac{\widetilde{J}}{\widetilde{\chi}_{I}}, & \widetilde{A}_{\infty}=-\frac{q_{1} \gamma_{5} t_{\infty}^{1}+q_{5} \gamma_{1} t_{\infty}^{5}}{k_{\infty}^{p}} \\
\widetilde{w}_{I \infty} & =-\frac{\left|\epsilon_{I J K}\right|}{2}\left[q k_{\infty}^{I} t_{\infty}^{J} t_{\infty}^{K}+2 q_{J} \gamma_{K}+\gamma_{J} \gamma_{K} \sum k_{\infty}^{L} q_{L}\right] .
\end{array}
$$

The expressions of $\widetilde{w}_{I \infty}^{(0)}$ and $\widetilde{A}_{\infty}^{(0)}$ remain complicated functions of the charges of the initial solution and the interested reader can easily compute them from (3.20) and (3.21). One can check by curiosity that the asymptotic value of the quartic invariant $\mathcal{I}_{4 \infty}(3.11)$ is indeed preserved

$$
\mathcal{I}_{4 \infty}=q q_{1} q_{5} q_{p}=\widetilde{q} \widetilde{q}_{1} \widetilde{q}_{5} \widetilde{q}_{p}-\widetilde{q}^{2} \widetilde{J}^{2}=\widetilde{\mathcal{I}}_{4 \infty} .
$$

By inserting (4.2) in the spectrally flowed six-dimensional metric (3.28), the $\mathrm{WAdS}_{3} \times \mathrm{SqS}^{3}$ asymptotic expansion of the metric is explicit

$$
\begin{aligned}
d s_{\infty}^{2}=\frac{\kappa^{2}}{4}[ & -r^{2} d \tau^{2}+\frac{d r^{2}}{r^{2}}+\gamma\left(d y_{\infty}+r d \tau\right)^{2}+\gamma\left(d \psi_{\infty}+\cos \theta d \phi\right)^{2} \\
& \left.+2 \alpha\left(d y_{\infty}+r d \tau\right)\left(d \psi_{\infty}+\cos \theta d \phi\right)+d \theta^{2}+\sin ^{2} \theta d \phi^{2}\right]+\ldots
\end{aligned}
$$

where we have defined the six-dimensional coordinates at infinity $\left(\tau, r, \theta, \phi, \psi_{\infty}, y_{\infty}\right)$ using the initial coordinates $(t, r, \theta, \phi, \psi, y)$ as follows

$$
\begin{aligned}
& y_{\infty} \equiv \sqrt{\mathcal{I}_{4 \infty}} \widetilde{\chi}_{p} \frac{\widetilde{w}_{p \infty}\left(\psi+\widetilde{A}_{\infty}^{(0)} \phi\right)-\widetilde{A}_{\infty}\left(y+\widetilde{w}_{p \infty}^{(0)} \phi\right)}{\widetilde{A}_{\infty}\left(\mathcal{I}_{4 \infty}-\widetilde{q}^{2} \widetilde{\chi}_{p} \widetilde{k}_{\infty}^{p} \widetilde{J}\right)-\widetilde{w}_{p \infty} \widetilde{q}^{2} \widetilde{\chi}_{p} \widetilde{J}}, \quad \tau \equiv \frac{t}{\sqrt{\mathcal{I}_{4 \infty}}}, \\
& \psi_{\infty} \equiv \frac{\left(\psi+\widetilde{A}_{\infty}^{(0)} \phi\right)\left(\mathcal{I}_{4 \infty}-\widetilde{q}^{2} \widetilde{\chi}_{p} \widetilde{k}_{\infty}^{p} \widetilde{J}\right)-\widetilde{q}^{2} \widetilde{\chi}_{p} \widetilde{J}\left(y+\widetilde{w}_{p \infty}^{(0)} \phi\right)}{\widetilde{A}_{\infty}\left(\mathcal{I}_{4 \infty}-\widetilde{q}^{2} \widetilde{\chi}_{p} \widetilde{k}_{\infty}^{p} \widetilde{J}\right)-\widetilde{w}_{p \infty} \widetilde{q}^{2} \widetilde{\chi}_{p} \widetilde{J}}
\end{aligned}
$$

\footnotetext{
${ }^{6}$ We use $t_{\infty}^{p}=1+k k_{\infty}^{p} \gamma_{p}=0$
} 
and where the warp constant factors, $\gamma$ and $\alpha$, and the length, $\kappa$, are given by

$$
\begin{aligned}
\gamma & =\left(\frac{\widetilde{q}_{p}}{\widetilde{w}_{p}}\right)^{2}\left[1+\frac{\widetilde{q} \widetilde{J}^{2}}{\widetilde{q}_{1} \widetilde{q}_{5} \widetilde{q}_{p}}\left(\left(\frac{\widetilde{w}_{p}}{\widetilde{q}_{p}}\right)^{2}-1\right)\right], \\
\alpha & =-\frac{\sqrt{\mathcal{I}_{4 \infty}}}{\widetilde{q}^{3} \widetilde{q}_{1} \widetilde{q}_{5} \widetilde{q}_{p}}\left(\widetilde{A}_{\infty}\left(\widetilde{k}_{\infty}^{p} \widetilde{q}_{p}^{2}+\widetilde{\chi}_{p} \widetilde{J}\right)+\widetilde{q}_{p}^{2} \widetilde{w}_{p \infty}\right), \\
\kappa^{2} & =4 \widetilde{q} \sqrt{\widetilde{q}_{1} \widetilde{q}_{5}} .
\end{aligned}
$$

The last condition to obtain $\mathrm{WAdS}_{3} \times \mathrm{SqS}^{3}$ in the asymptotic region is on the periods for $\left(y_{\infty}, \psi_{\infty}, \phi\right)$

$$
\left(y_{\infty}, \psi_{\infty}, \phi\right)=\left\{\begin{array}{l}
\left(y_{\infty}, \psi_{\infty}, \phi\right)+2 \pi\left(T_{y},-T_{\psi}, 0\right) \\
\left(y_{\infty}, \psi_{\infty}, \phi\right)+2 \pi(0,2,0) \\
\left(y_{\infty}, \psi_{\infty}, \phi\right)+2 \pi(0,1,1)
\end{array} .\right.
$$

Such periodicities are complicated to obtain while keeping the usual periods for $(y, \psi, \phi)$

$$
y=y+2 \pi, \quad \psi=\psi+4 \pi, \quad(\psi, \phi)=(\psi, \phi)+(2 \pi, 2 \pi) .
$$

However, one can just reverse the perspective by imposing directly the periods (4.9) for $\left(y_{\infty}, \psi_{\infty}, \phi\right)$ and express the corresponding periods of $(y, \psi, \phi)$ by inverting (4.7). This has the advantage of adding no new complicated constraints on the parameters of the solution but the main drawback is that this drastic modification of periods of $(y, \psi, \phi)$ can induce conical singularities in the IR wherever the $\mathrm{S}^{3}$ shrinks.

\subsection{The infrared geometry}

Generalized spectral flows preserve the bubbling feature of the initial solution: the number of centers and their positions on the $\mathbb{R}^{3}$ base space are straightforwardly preserved. They also preserve all the conditions for the absence of closed timelike curves as detailed in section 3.3. Moreover, they transform a singular supertube center to a smooth GibbonsHawking center. ${ }^{7}$ A series expansion of the spectrally flowed solution around the center $J$ (where $J=0,1,5, p$ ) gives

$$
\begin{array}{rlrl}
\widetilde{Z}_{I} \sim \widetilde{z}_{I J}, & \widetilde{V} \sim \frac{\widetilde{q}_{J}}{r_{J}}, & \widetilde{\mu} \sim \widetilde{\mu}_{J} r_{J}, \quad \widetilde{W}_{I} \sim \widetilde{\chi}_{I J}, & \widetilde{P}_{I} \sim \widetilde{k}_{I J} \\
\widetilde{A} \sim\left(\widetilde{A}_{J}^{(0)}+\widetilde{A}_{J} \cos \theta_{J}\right) d \phi, & \widetilde{w}_{I} \sim\left(\widetilde{w}_{I J}^{(0)}+\widetilde{w}_{I J} \cos \theta_{J}\right) d \phi, \quad r_{J} \rightarrow 0,
\end{array}
$$

where the tilded quantities in the right-hand sides are constant. It is not necessary for what will follow to write their complicated dependence on the parameters of the initial solution. ${ }^{8}$ The three noteworthy points are

\footnotetext{
${ }^{7} \mathrm{~A}$ two-charge supertube is regular only when its magnetic dipole charge sources the $\mathrm{P}$ charge of the system. In our convention, this is a two-charge supertube of type p. Singular supertube centers are supertubes which source magnetically the D1 or the D5 charges [44].

${ }^{8}$ For the interested reader, they can be easily derived with a calculation software using the transformation rules (3.16) on the initial almost-BPS supertube solution given in (3.18), (3.20) and (3.21) and then taking the limit $r_{J} \rightarrow 0$.
} 
- The ratio $\frac{\widetilde{A}_{J}}{\widetilde{q}_{J}}$ is equal to 1 . This is a key feature of an ambipolar Gibbons-Hawking metric. Indeed, in a generic Gibbons-Hawking metric (3.2), the term proportional to $\cos \theta d \phi$ in $\frac{A}{V}$ must be exactly $\cos \theta d \phi$.

- The quantity $\widetilde{k}_{p J} \widetilde{A}_{J}+\widetilde{w}_{p J}$ is equal to zero. Thus, the $\mathrm{U}(1)$ fiber defined by $\widetilde{A}_{p}+d y$ in (3.15) has no term proportional to $\cos \theta_{J} d \phi$ when we approach the center $J$. The local five-dimensional base space is then an exact direct product of a $S^{1}$ with a Gibbons-Hawking space.

- All the quantities in (4.11) except $\widetilde{z}_{I J}$ are rational functions of the initial parameters. This will be an important ingredient if we require the local geometry to be a discrete quotient of $\mathrm{S}^{1} \times \mathbb{R}^{4}$.

We can now use the expansions (4.11) and the three remarks above to compute the limit of the spectrally flowed six-dimensional metric around the center $J$ :

$$
\begin{aligned}
d s_{J}^{2}=\widetilde{q}_{J} \sqrt{\widetilde{z}_{1 J} \widetilde{z}_{5 J}}[ & -d \tau_{J}^{2}+\frac{d r_{J}^{2}}{r_{J}}+r_{J}\left(\left(d \psi_{J}+\left(1+\cos \theta_{J}\right) d \phi\right)^{2}+d \theta_{J}^{2}+\sin ^{2} \theta_{J} d \phi^{2}\right) \\
& \left.+\frac{\widetilde{z}_{p J}}{\widetilde{q}_{J} \widetilde{z}_{1 J} \widetilde{z}_{5 J}} d y_{J}^{2}\right]
\end{aligned}
$$

where we have defined the six-dimensional Gibbons-Hawking coordinate system $\left(\tau_{J}, r_{J}, \theta_{J}, \psi_{J}, \phi, y_{J}\right)$ as a function of the initial coordinates $(t, r, \theta, \phi, \psi, y)$ :

$$
\begin{aligned}
\tau_{J} \equiv \frac{t}{\sqrt{\widetilde{q}_{J} \widetilde{z}_{1 J} \widetilde{z}_{5 J} \widetilde{z}_{p J}}}, & r_{J} \equiv \sqrt{r^{2}+a_{J}^{2}-2 a_{J} r \cos \theta}, \quad \cos \theta_{J} \equiv \frac{r \cos \theta-a_{J}}{r_{J}} \\
\psi_{J} \equiv \frac{\psi+\left(\widetilde{A}_{J}^{(0)}-1\right) \phi}{\widetilde{q}_{J}}, & y_{J} \equiv y-\frac{t}{\widetilde{\chi}_{p J}}+\widetilde{k}_{p J} \psi+\left(\widetilde{k}_{p J} \widetilde{A}_{J}^{(0)}+\widetilde{w}_{p J}^{(0)}\right) \phi
\end{aligned}
$$

We recognize the metric of a U(1) fiber over a Gibbons-Hawking space. Thus, the local geometry has no curvature singularity. However, a conical singularity can occur depending on the periodicities of $\left(y_{J}, \psi_{J}, \phi\right)$. If the periodicities were the usual Gibbons-Hawking periods (4.10), the base space would be a discrete $\mathbb{Z}_{\left|\widetilde{q}_{J}\right|}$ quotient of $S^{1} \times \mathbb{R}^{4}$. The absence of conical singularity at $r_{J}=0$ would simply require that $\widetilde{q}_{J}$ is integer-valued and would impose some arithmetic constraints on the coefficients involved in (4.13). ${ }^{9}$ However, the modification of the periodicities at infinity have drastically modified the periods of $(y, \psi, \phi)$ and the smoothness analysis will require the full mathematical machinery which we briefly detail following [14, 46, 47].

Let us first map the Gibbons-Hawking patch of angles $\left(\theta_{J}, \psi_{J}, \phi\right)$ to the $\mathrm{S}^{3}$ patch $\left(\theta_{J}, \phi_{L J}, \phi_{R J}\right)$ by taking

$$
\phi_{R J}=\frac{\psi_{J}}{2}, \quad \phi_{L J}=\frac{\psi_{J}}{2}+\phi .
$$

\footnotetext{
${ }^{9}$ See $[12]$ for examples of this kind.
} 
The spacelike components of the metric $\left(d t_{J}=0\right)$ gives the spherically symmetric metric on $\mathrm{S}^{1} \times \mathbb{R}^{4}$

$$
\begin{aligned}
\frac{1}{\widetilde{q}_{J} \sqrt{\widetilde{z}_{1 J} \widetilde{z}_{5 J}}} d s_{a}^{2}= & \frac{d r_{J}^{2}}{r_{J}}+\frac{\widetilde{z}_{p J}}{\widetilde{q}_{J} \widetilde{z}_{1 J} \widetilde{z}_{5 J}} d y_{J}^{2} \\
& +r_{J}\left[d \theta_{J}^{2}+2\left(1+\cos \theta_{J}\right) d \phi_{L J}^{2}+2\left(1-\cos \theta_{J}\right) d \phi_{R J}^{2}\right]
\end{aligned}
$$

The periodicities of $\left(y_{J}, \phi_{L J}, \phi_{R J}\right)$ can be read off from the periodicities of $\left(y_{\infty}, \psi_{\infty}, \phi\right)(4.9)$ with the sequence of three linear changes of coordinates $\left(y_{\infty}, \psi_{\infty}, \phi\right) \rightarrow(y, \psi, \phi)$ in (4.7), $(y, \psi, \phi) \rightarrow\left(y_{J}, \psi_{J}, \phi\right)$ in $(4.13)$ and $\left(y_{J}, \psi_{J}, \phi\right) \rightarrow\left(y_{J}, \phi_{L J}, \phi_{R J}\right)$ in (4.14). After few lines of computation, the periodicities translate into the following identifications ${ }^{10}$

$$
\left(y_{J}, \phi_{L J}, \phi_{R J}\right)= \begin{cases}\left(y_{J}, \phi_{L J}, \phi_{R J}\right)+2 \pi\left(\alpha_{A}, \beta_{A}, \beta_{A}\right) & (A) \\ \left(y_{J}, \phi_{L J}, \phi_{R J}\right)+2 \pi\left(\alpha_{B}, \beta_{B}, \beta_{B}\right) & (B) \\ \left(y_{J}, \phi_{L J}, \phi_{R J}\right)+2 \pi\left(\alpha_{C}, 1+\beta_{C}, \beta_{C}\right) & (C)\end{cases}
$$

where the coefficients $\alpha_{i}$ and $\beta_{i}$ are complicated but computable rational functions depending on:

- The parameters of the initial almost-BPS solution.

- The spectral flow parameters $\gamma_{I}$.

- The periods $T_{y}$ and $T_{\psi}$ of the angles of the UV $\mathrm{WAdS}_{3}(2.7)$.

- The square root of the asymptotic value of the quartic invariant $\sqrt{\mathcal{I}_{4 \infty}}$ (4.5). The local geometry is a discrete quotient of $\mathrm{S}^{1} \times \mathbb{R}^{4}$ if $\alpha_{J}$ and $\beta_{J}$ are rational numbers. Thus, all the initial parameters and $\sqrt{\mathcal{I}_{4 \infty}}$ must be rational. ${ }^{11}$ Choosing the other parameters to be rational is easy. However, imposing $\sqrt{\mathcal{I}_{4 \infty}}$ to be rational requires a little bit of arithmetic.

Conical singularities only occur at points that are invariant under the operation

$$
A^{n_{A}} B^{n_{B}} C^{n_{C}}, \quad\left(n_{A}, n_{b}, n_{c}\right) \in \mathbb{Z} .
$$

Furthermore, they all arise at $r_{J}=0$ where $\phi_{L J}$ and $\phi_{R J}$ are both degenerate, at $\theta_{J}=0$ where $\phi_{R J}$ is degenerate and at $\theta_{J}=\pi$ where $\phi_{L J}$ is degenerate. The periods of $\phi_{R J}$ and $\phi_{L J}$ are almost identical with a difference of $2 \pi$ for the periodicity $C$, so if the identifications (4.16) at $r_{J}=0$ do not destroy smoothness, they will also ensure the absence of singularities at $\theta_{J}=0$ or $\pi$.

At $r_{J}=0$, in order for the shifts $\phi_{L J} \rightarrow \phi_{L J}+2 \pi$ and $\phi_{R J} \rightarrow \phi_{R J}+2 \pi$ at fixed $y_{J}$ to be a closed orbit, any triplet of integers $\left(n_{A}, n_{B}, n_{C}\right)$ where $y_{J} \rightarrow y_{J}$ under (4.17) must satisfy $n_{A} \beta_{A}+n_{B} \beta_{B}+n_{C} \beta_{C} \in \mathbb{Z}$. In more concrete terms, any operation (4.17) which leaves $y_{J}$ invariant, that is to say where $n_{A} \alpha_{A}+n_{B} \alpha_{B}+n_{C} \alpha_{C}=0$, must transform

\footnotetext{
${ }^{10}$ For readability, we have dropped the index $J$ referring to the center but the coefficient $\alpha_{i}$ and $\beta_{i}$ are not identical for the four centers.

${ }^{11}$ This means that the entropy of the corresponding black hole given by $S=2 \pi \sqrt{\mathcal{I}_{4 \infty}}$ belongs to $2 \pi \mathbb{Q}$.
} 
$\phi_{L J} \rightarrow \phi_{L J}+2 \pi N$ and $\phi_{L J} \rightarrow \phi_{L J}+2 \pi N^{\prime}$ where $N$ and $N^{\prime}$ are both integers. Using simple arithmetic arguments one can show that this is equivalent to prove the condition for the three sets of integers $\left(0, n_{B}, n_{C}\right),\left(n_{A}, 0, n_{C}\right)$ and $\left(n_{A}, n_{B}, 0\right)$. If the conditions are satisfied for each set, the action of the quotient is free and the local geometry around the center $J$ is then a smooth discrete quotient of $S^{1} \times \mathbb{R}^{4}$.

This analysis applies at every center. The total number of smoothness conditions is then $12(3 \times 4$ centers $)$. The number of parameters is still greater than the number of conditions which gives good hope to draw a systematic construction procedure.

\subsection{The construction procedure}

We sketch briefly a technical summary of what we have done until now to build asymptotically $\mathrm{WAdS}_{3} \times \mathrm{SqS}^{3}$ geometries:

- We start with the family of almost-BPS four-center solutions of three supertubes in $\mathbb{R}^{4}$. Initially, it is a family of 15 rational parameters: $q, Q_{I}^{(J)}, \kappa^{I}, a_{I}$ and $k_{\infty}^{I}$. The regularity of the solution imposes three bubble equations (3.24), the condition on the asymptotics requires $j_{R}=0(3.28)$ and the positivity of the quartic invariant $\mathcal{I}_{4}$ is satisfied by imposing all the initial charges and dipole charges to be positive except one. Furthermore, $\sqrt{\mathcal{I}_{4 \infty}}$ needs to be a rational number which is not an equation but one can consider that this fixes a parameter. We have consequently a 10-parameter family of initial almost-BPS solutions.

- After three generalized spectral flows, we have three new parameters $\gamma_{I}$ whose one is fixed to have rational spectrally flowed charges. Moreover, the periods $T_{y}$ and $T_{\psi}$ of the angles of the $\mathrm{WAdS}_{3} \times \mathrm{SqS}^{3}$ region can also be considered as free parameters.

- We have in total a 14-parameter family of bubbling asymptotically $\mathrm{WAdS}_{3} \times \mathrm{SqS}^{3}$ geometries. The smoothness of the geometry in the IR requires 12 arithmetic conditions as discussed in the previous section. These conditions do not exactly fix parameters so the parameter space of the resulting family of smooth solutions is complicated to define. However, many solutions can be easily generated by generating parameters and by checking for each set of parameters if the 12 arithmetic conditions can be satisfied. We give an example of such a solution in the next section.

\subsection{An explicit example}

We construct an explicit example of the procedure discussed above. We picked an almostBPS three-supertube solution in $\mathbb{R}^{4}$ giving the solution which we use in the first step of the procedure:

$$
\begin{aligned}
& q=\Lambda, \quad \kappa_{1}=\frac{\Lambda}{2}, \quad \kappa_{5}=-\frac{2 \Lambda}{3}, \quad \kappa_{p}=\frac{\Lambda}{2}, \quad Q_{5}^{(1)}=\Lambda, \\
& Q_{p}^{(1)}=\frac{2 \Lambda}{3}, \quad Q_{1}^{(5)}=\Lambda, \quad Q_{p}^{(5)}=\Lambda, \quad Q_{1}^{(p)}=\frac{\Lambda}{3}, \quad Q_{5}^{(p)}=\frac{4 \Lambda}{3},
\end{aligned}
$$

where $\Lambda \in \mathbb{Q}^{+}$is a degree of freedom of the charges which does not compromise the regularity of the solution and the condition on the asymptotics. We can consider $\Lambda$ as a 
free parameter all along the construction. The coordinates of the three supertube centers on the z-axis are

$$
a_{1}=1, \quad a_{5}=\frac{36}{13}, \quad a_{p}=24 .
$$

The solution is asymptotically $\mathrm{AdS}_{2} \times \mathrm{S}^{1} \times \mathrm{S}^{3}$ which implies that the center positions are scaling invariant $a_{I} \rightarrow \lambda a_{I}$ [9]. Consequently, one can freely rescale (4.19) to make the inter-center distances as small as we want.

We did not fix yet the constant terms $k_{\infty}^{I}$ since they are not involved in the regularity of the solution. They are actually irrelevant from the point of view of the initial almostBPS solution since they affect only the asymptotic values of the gauge, $A_{I}$, of the solution which can be gauge-fixed to zero. However, they affect greatly the solutions one obtains after spectral flows.

From (3.18), one can derive the asymptotic values of the D1, D5, P charges of the initial solution, the left and right angular momenta and the entropy of the corresponding three-charge black hole

$$
\begin{aligned}
q_{1} & =\frac{4 \Lambda}{3}, \quad q_{5}=\frac{9 \Lambda}{4}, \quad q_{p}=\frac{4 \Lambda}{3}, \\
j_{L} & =j_{R}=0, \\
S & =2 \pi \sqrt{\mathcal{I}_{4 \infty}}=4 \pi \Lambda^{2} .
\end{aligned}
$$

One can now play with the spectral flow parameters $\gamma_{I}$ and the constants $k_{\infty}^{I}$ to generate an extremal non-supersymmetric smooth asymptotically $\mathrm{WAdS}_{3} \times \mathrm{SqS}^{3}$ bubbling geometry. We found an infinite number of such solutions. To give an example, we pick one of these solutions:

$$
\gamma_{1}=\frac{1}{2}, \quad \gamma_{5}=-1, \quad \gamma_{p}=-1, \quad k_{\infty}^{1}=-\frac{3}{2}, \quad k_{\infty}^{5}=3, \quad k_{\infty}^{p}=1 .
$$

We can derive the full geometry by computing the metric and the gauge fields (3.15) and (3.16). We will just focus on the $\mathrm{WAdS}_{3} \times \mathrm{SqS}^{3}$ asymptotic region which is given by

$$
\begin{aligned}
d s_{\infty}^{2}=\frac{1}{3} \sqrt{\frac{485}{6}}[ & -r^{2} d \tau^{2}+\frac{d r^{2}}{r^{2}}+\frac{701}{485}\left(d y_{\infty}+r d \tau\right)^{2}+\frac{701}{485}\left(d \psi_{\infty}+\cos \theta d \phi\right)^{2} \\
& \left.+\frac{1302}{485}\left(d y_{\infty}+r d \tau\right)\left(d \psi_{\infty}+\cos \theta d \phi\right)+d \theta^{2}+\sin ^{2} \theta d \phi^{2}\right] .
\end{aligned}
$$

We choose the periodicities of the angles to be ${ }^{12}$

$$
\left(y_{\infty}, \psi_{\infty}, \phi\right)=\left\{\begin{array}{l}
\left(y_{\infty}, \psi_{\infty}, \phi\right)+2 \pi\left(\frac{24}{43} T,-\frac{8}{11} T, 0\right) \\
\left(y_{\infty}, \psi_{\infty}, \phi\right)+2 \pi(0,2,0) \\
\left(y_{\infty}, \psi_{\infty}, \phi\right)+2 \pi(0,1,1)
\end{array},\right.
$$

\footnotetext{
${ }^{12}$ Many other possibilities were available.
} 
where $T$ is a free parameter. For the reader interested in the smoothness of the bubbling geometry in the IR, the metric and the periodicities of the angles around the centers are given in the appendix B. We found that the IR bubbling geometry is smooth if and only if $T=\frac{a}{b} \in \mathbb{Q}$ and $b$ is not divisible by 2 or 13 .

\section{$5 \quad$ Asymptotically NHEK bubbling geometries}

In the previous section, we have constructed in detail a large family of extremal nonsupersymmetric bubbling solutions which cap off smoothly in the IR and which are asymptotically $\mathrm{WAdS}_{3} \times \mathrm{SqS}^{3}$. In the present section, we push a bit further the construction to asymptotically NHEK bubbling solutions. The path from $\mathrm{WAdS}_{3} \times \mathrm{SqS}^{3}$ to NHEK requires to relate the $\mathrm{WAdS}_{3}$ region of our solutions to the near-horizon region of the over-rotating 5d D1-D5-P black hole detailed in section 2.1. This essentially means that we have to express the charges, angular momentum and mass of the D1-D5-P black hole in terms of the parameters of our solutions. Once this is done, we have to impose the NHEK periodicities of the angles at infinity (2.8) and check the smoothness of the IR geometry as it has been done for asymptotically $\mathrm{WAdS}_{3}$ geometries in section 4.2. At first sight, this might seem to be a mere formality. However, the fact that the periods of $y_{\infty}$ and $\psi_{\infty}$ were free parameters for asymptotically $\mathrm{WAdS}_{3}$ solutions was practical to satisfy the twelve conditions of smoothness at the centers. Now that the periods are connected to the parameters defining the bubbling geometry, this requires more work.

In this section, we use all the results obtained in the previous section. We have started with the family of almost-BPS three-supertube solutions in $\mathbb{R}^{4}$ and performed the sequence of generalized spectral flows detailed in section 4.1 to obtain an asymptotically $\mathrm{WAdS}_{3} \times \mathrm{SqS}^{3}$ bubbling geometry. In section 5.1 we will match this asymptotic region to a near-horizon region of an extremal non-supersymmetric D1-D5-P black hole. We will identify the corresponding periodicities and see how such solutions can be systematically generated in section 5.2. At the end of the section, we will give an explicit example of a solution.

\subsection{Matching the WAdS $_{3}$ UV geometry to NHEK}

After applying the sequence of generalized spectral flows to our family of almost-BPS solutions, the asymptotic metric is given by (4.6) where the constant warp factors $\gamma$ and $\alpha$ and the length $\kappa$ are defined in (4.8). We want to relate this geometry to the nearhorizon geometry of an extremal non-supersymmetric D1-D5-P black hole determined by four parameters $a, \delta_{1}, \delta_{5}$ and $\delta_{p}$ and given by the metric (2.9) where $\gamma, \alpha$ and $\kappa$ are defined by (2.10). We use the three identities between $\gamma, \alpha$ and $\kappa$ to relate $a, \delta_{1}$ and $\delta_{5}$ to the parameters of our solutions and we use the matching of the entropy to find $\delta_{p}$. After few 
lines of computation, we obtain

$$
\begin{aligned}
a & =2\left(\mathcal{I}_{4 \infty} \gamma_{1} \gamma_{5} t_{\infty}^{1} t_{\infty}^{5}\right)^{1 / 4}, \\
s_{1} & =\frac{1}{2}\left[\frac{q q_{1} t_{\infty}^{1}{ }^{2}+\gamma_{1}\left(\gamma_{1} q_{5} q_{p}-2 \sqrt{\mathcal{I}_{4 \infty}} t_{\infty}^{1}\right)}{\gamma_{1} t_{\infty}^{1} \sqrt{\mathcal{I}_{4 \infty}}}\right]^{1 / 2}, \\
s_{5} & =\frac{1}{2}\left[\frac{q q_{5} t_{\infty}^{5}{ }^{2}+\gamma_{5}\left(\gamma_{5} q_{1} q_{p}-2 \sqrt{\mathcal{I}_{4 \infty}} t_{\infty}^{5}\right)}{\gamma_{5} t_{\infty}^{5} \sqrt{\mathcal{I}_{4 \infty}}}\right]^{1 / 2}, \\
s_{p} & =\frac{\mathcal{I}_{4 \infty}}{4 a^{3}} \frac{s_{1} s_{5}+c_{1} c_{5} \sqrt{\mathcal{H}}}{c_{1}^{2} c_{5}^{2}-s_{1}^{2} s_{5}^{2}},
\end{aligned}
$$

where $s_{I}=\sinh \delta_{I}, c_{I}=\cosh \delta_{I}$ and $\mathcal{H}$ is defined as

$$
\begin{aligned}
\mathcal{H} & \equiv 1-16 \frac{a^{6}\left(c_{1}^{2} c_{5}^{2}-s_{1}^{2} s_{5}^{2}\right)}{\mathcal{I}_{4 \infty}} \\
& =1-256 \sqrt{\gamma_{1} \gamma_{5} t_{\infty}^{1} t_{\infty}^{5}}\left(\gamma_{1} q_{5} t_{\infty}^{5}+\gamma_{5} q_{1} t_{\infty}^{1}\right)\left(q t_{\infty}^{1} t_{\infty}^{5}+q_{p} \gamma_{1} \gamma_{5}\right) .
\end{aligned}
$$

The mass, the D1, D5 and P charges and the left angular momentum of the corresponding Cvetic-Youm black hole can be derived using (2.1). However, the most interesting quantities are the NHEK periods $T_{y}$ and $T_{\psi}(2.8)$. Using (5.1), we can show that they are rational numbers if:

- $\sqrt{\mathcal{I}_{4 \infty}}$ is rational. This is the same condition as the one imposed for asymptotically $\mathrm{WAdS}_{3}$ bubbling geometries.

- $\sqrt{\mathcal{H}}$ is rational. This is a more complicated condition to satisfy than the previous one. Tricky arithmetic is required.

Once the matching to NHEK is performed, one can look at the IR bubbling region of our solutions. The periodicities of the angles around each center depends on $T_{y}$ and $T_{\psi}$. Thus the local geometries are quotients of $\mathbb{R}^{4} \times \mathrm{S}^{1}$ only if they are rational. Moreover, conical singularities might still occur as for asymptotically $\mathrm{WAdS}_{3}$ bubbling geometries. We use the same smoothness analysis as in section 4.1 to derive 12 conditions to have smooth discrete quotients on $\mathbb{R}^{4} \times \mathrm{S}^{1}$ around the centers.

\subsection{The construction procedure}

The procedure to build smooth asymptotically NHEK bubbling geometries is similar to the one depicted in section 4.3:

- We start with the family of almost-BPS four-center solutions of three supertubes in $\mathbb{R}^{4}$. Initially, it is a family parametrized by 15 rational parameters: $q, Q_{I}^{(J)}, \kappa^{I}, a_{I}$ and $k_{\infty}^{I}$. We solve the three bubble equations $(3.24), j_{R}=0(3.28)$ and we require the positivity of the quartic invariant $\mathcal{I}_{4}$ by imposing all the initial charges to be positive except one. Furthermore, $\sqrt{\mathcal{I}_{4 \infty}}$ needs to be a rational number which fixes a parameter. We have consequently a 10-parameter family of initial almost-BPS solutions. 
- After three generalized spectral flows, we have two more parameters $\gamma_{I}\left(\gamma_{p}\right.$ is fixed to have rational spectrally flowed charges). The condition on the periods $T_{y}$ and $T_{\psi}$ to be rational requires some arithmetic machinery which fixes 3 parameters (the two remaining spectral flow parameters and $\left.k_{\infty}^{5}\right)$.

- We have in total an 8-parameter family of bubbling asymptotically $\mathrm{NHEK}_{3}$ geometries. The smoothness of the geometry in the IR requires 12 arithmetic conditions. Even if the parameter space is not easy to determine, we can perform a loop generating technique to build a large number of such solutions. We give an example of such a solution in the next section.

\subsection{An explicit example}

We construct an explicit example of the procedure discussed above. We choose a smooth almost-BPS three-supertube solution in $\mathbb{R}^{4}$ satisfying the first point of the procedure:

$$
\begin{aligned}
& q=1, \quad \kappa_{1}=\Lambda, \quad \kappa_{5}=-\frac{\Lambda}{2}, \quad \kappa_{p}=\frac{\Lambda}{3}, \quad Q_{5}^{(1)}=\Lambda^{2}, \\
& Q_{p}^{(1)}=\frac{\Lambda^{2}}{2}, \quad Q_{1}^{(5)}=\frac{2 \Lambda^{2}}{3}, \quad Q_{p}^{(5)}=\frac{\Lambda^{2}}{3}, \quad Q_{1}^{(p)}=\frac{\Lambda^{2}}{2}, \quad Q_{5}^{(p)}=\Lambda^{2},
\end{aligned}
$$

where $\Lambda \in \mathbb{Q}^{+}$corresponds to the charge-scaling free parameter. We choose a slightly different charge-scaling $\Lambda$ than in section 4.4. They are actually equivalent. The present choice is just more adapted to the matching with NHEK. The coordinates of the three supertube centers on the z-axis are

$$
a_{1}=1, \quad a_{5}=\frac{3}{14}(17+\sqrt{65}), \quad a_{p}=\frac{3}{4}(9+\sqrt{65}) .
$$

Once again, the $\mathrm{AdS}_{2} \times \mathrm{S}_{1} \times \mathrm{S}^{3}$ asymptotics of the solution allows us to rescale $a_{I} \rightarrow \lambda a_{I}$ as small as we want. The irrationality of the inter-center distances does not impact the smoothness of the solution around the centers.

From (3.18), one can derive the asymptotic values of the initial D1, D5, P charges of the solution, the left and right angular momenta and the entropy of the initial system

$$
\begin{aligned}
q_{1} & =\frac{4 \Lambda^{2}}{3}, \quad q_{5}=\frac{4 \Lambda^{2}}{3}, \quad q_{p}=\Lambda^{2}, \\
j_{L} & =j_{R}=0, \\
S & =2 \pi \sqrt{\mathcal{I}_{4 \infty}}=\frac{8 \pi}{3} \Lambda^{3} .
\end{aligned}
$$

One can now play with the spectral flow parameters $\gamma_{I}$ and the constants $k_{\infty}^{I}$ to generate an extremal non-supersymmetric smooth asymptotically $\mathrm{NHEK}_{3} \times \mathrm{SqS}^{3}$ bubbling geometry. This requires $\sqrt{\mathcal{H}}$ in $(5.2)$ to be rational. After a rather technical arithmetic computation we found several values for $\gamma_{1}, \gamma_{5}$ and $k_{\infty}^{5}$ which lead to rational NHEK periods $T_{y}$ and $T_{\psi}$ without inducing any conical singularities at the centers:

$$
\begin{aligned}
& \gamma_{1}=\frac{1}{3 \Lambda-2}, \quad \gamma_{5}=\frac{9 \Lambda-6}{40 \Lambda^{2}}, \quad \gamma_{p}=-1, \\
& k_{\infty}^{1}=2, \quad k_{\infty}^{5}=-\frac{\Lambda}{3} \frac{2+37 \Lambda}{3 \Lambda-2}, \quad k_{\infty}^{p}=1 .
\end{aligned}
$$


We can derive the full geometry by computing the metric and the gauge fields (3.15) and (3.16). We will just focus on the NHEK asymptotic region which is given by

$$
\begin{aligned}
d s_{\infty}^{2}=\frac{\Lambda^{2}}{3}[ & -r^{2} d \tau^{2}+\frac{d r^{2}}{r^{2}}+\frac{34}{25}\left(d y_{\infty}+r d \tau\right)^{2}+\frac{34}{25}\left(d \psi_{\infty}+\cos \theta d \phi\right)^{2} \\
& \left.+\frac{12}{5}\left(d y_{\infty}+r d \tau\right)\left(d \psi_{\infty}+\cos \theta d \phi\right)+d \theta^{2}+\sin ^{2} \theta d \phi^{2}\right] .
\end{aligned}
$$

The NHEK angle periodicities are

$$
\left(y_{\infty}, \psi_{\infty}, \phi\right)=\left\{\begin{array}{l}
\left(y_{\infty}, \psi_{\infty}, \phi\right)+2 \pi\left(\frac{75}{32 \Lambda},-\frac{93}{32 \Lambda}, 0\right) \\
\left(y_{\infty}, \psi_{\infty}, \phi\right)+2 \pi(0,2,0) \\
\left(y_{\infty}, \psi_{\infty}, \phi\right)+2 \pi(0,1,1)
\end{array} .\right.
$$

The asymptotic NHEK region corresponds to the near-horizon region of an extremal Kerr black hole given by the following mass, angular momenta and charges:

$$
\begin{aligned}
M & =\frac{287 \Lambda^{2}}{75}, \\
J_{R} & =0, \quad J_{L}=\frac{124 \Lambda^{3}}{75}, \\
Q_{1} & =\frac{16 \Lambda^{2}}{15}, \quad Q_{5}=\frac{16 \Lambda^{2}}{15}, \quad Q_{p}=\frac{21 \Lambda^{2}}{25}
\end{aligned}
$$

For the reader interested in the feature of the bubbling geometry in the IR, we gave the local metrics and the periodicities of the angles around the centers in the appendix C.

For any rational values of $\Lambda$, we found a smooth non-supersymmetric extremal geometry which is bubbling in the IR and NHEK in the UV.

One can also be interested in computing the RR three-form flux $F^{(3)}$ of the final solution. One can perform a similar computation of the spectrally flowed RR three-form flux $F^{(3)}$ as in [23].

\section{Conclusions}

In this paper, we have constructed a family of smooth bubbling solutions in six dimensions which are asymptotic to either generic $\mathrm{WAdS}_{3} \times \mathrm{SqS}^{3}$ or NHEK. We gave explicit examples of the construction which can be used for different purposes:

- One can investigate their CFT dual states. They can give some hints on the nature of the $\mathrm{CFT}_{2}$ dual to $\mathrm{WAdS}_{3}$ or the $\mathrm{CFT}_{2}$ dual to NHEK.

- Nearly extreme black hole have been seen in the sky [28]. From an astrophysical point of view, one can compute the Kerr multipole moments of our solutions to see if there exist deviations from the Kerr-Newman black hole solution. This could give interesting observable quantities in order to detect some imprints of the microstate structure of black holes in the gravitational wave emission after a collision of two black holes. 
Furthermore, one can extend the construction to have microstates of the whole KerrNewman black hole solution in five dimensions and not only its near-horizon geometry. In the context of multicenter solutions, we have to "bring back the ones" in the harmonic functions which will make the solutions to be asymptotically flat. This is the subject of future work.

\section{Acknowledgments}

I am grateful to Iosif Bena, Guillaume Bossard and Monica Guica for their useful advice. The work of PH was supported by a CDSN from ENS Lyon and by the ANR grant BlackdS-String ANR-16- CE31-0004-01.

\section{A Derivation of $v_{I}$ and $v_{0}$}

In this section, we derive the electromagnetic one-forms $v_{0}$ and $v_{I}$. They are involved in the generalized spectral flow transformations of an almost-BPS multicenter solutions (3.16). This is why they have to be derived in order to match the asymptotics of the spectrally flowed solutions to $\mathrm{WAdS}_{3}$ or NHEK and to regularize them. They satisfy the equations (3.10) where $Z_{I}, K_{I}$ and $V$ are given (3.17) and (3.18). We decompose the equations as follows

$$
\begin{aligned}
\star_{3} d v_{I}=\frac{\left|\epsilon_{I J K}\right|}{2}[ & -q k_{\infty}^{J} k_{\infty}^{K} \star_{3} d T^{(0)}+2 k_{\infty}^{J} \kappa^{K} \star_{3} d T_{J}^{(1)}-2 Q_{J}^{(I)} \star_{3} d T_{J}^{(2)} \\
& \left.-\frac{\kappa_{J} \kappa_{K}}{q} \star_{3} d T_{J K}^{(3)}\right], \\
\star_{3} d v_{0}=\frac{\left|\epsilon_{I J K}\right|}{6}[ & q k_{\infty}^{I} k_{\infty}^{J} k_{\infty}^{K} \star_{3} d T^{(0)}+6 k_{\infty}^{I} Q_{J}^{(I)} \star_{3} d T_{J}^{(1)}-3 k_{\infty}^{I} k_{\infty}^{J} \kappa_{K} \star_{3} d T_{K}^{(2)} \\
& \left.+6 \frac{\kappa_{I} Q_{J}^{(I)}}{q} \star_{3} d T_{I J}^{(3)}+3 \frac{k_{\infty}^{I} \kappa_{J} \kappa_{K}}{q} \star_{3} d T_{J K}^{(4)}+\frac{\kappa_{I} \kappa_{J} \kappa_{K}}{q^{2}} \star_{3} d T_{I J K}^{(5)}\right],
\end{aligned}
$$

where $T^{(0)}, T_{I}^{(2)}, T_{I J}^{(3)}, T_{I J}^{(4)}$ and $T_{I J K}^{(5)}$ satisfy

$$
\begin{aligned}
\star_{3} d T^{(0)}= & d\left(\frac{1}{r}\right), \quad \star_{3} d T_{I}^{(1)}=d\left(\frac{1}{r_{I}}\right), \quad \star_{3} d T_{I}^{(2)}=\frac{a_{I}}{r} d\left(\frac{1}{r_{I}}\right)-\frac{a_{I}}{r_{I}} d\left(\frac{1}{r}\right), \\
\star_{3} d T_{I J}^{(3)}= & \frac{a_{I}}{r_{I}} d\left(\frac{1}{r_{J}}\right)-\frac{a_{I}}{r_{J}} d\left(\frac{1}{r_{I}}\right), \quad \star_{3} d T_{I J}^{(4)}=\left(1-\frac{a_{I} a_{J}}{r^{2}}\right) d\left(\frac{r}{r_{I} r_{J}}\right), \\
\star_{3} d T_{I J K}^{(5)}= & \left(\frac{1}{a_{I} a_{J}}+\frac{1}{r^{2}}-\frac{1}{a_{I} a_{K}}-\frac{1}{a_{J} a_{K}}\right) \frac{r}{r_{I} r_{J}} d\left(\frac{1}{r_{K}}\right) \\
& +\left(\frac{1}{a_{I} a_{K}}+\frac{1}{r^{2}}-\frac{1}{a_{I} a_{J}}-\frac{1}{a_{J} a_{K}}\right) \frac{r}{r_{I} r_{K}} d\left(\frac{1}{r_{J}}\right) \\
& +\left(\frac{1}{a_{J} a_{K}}+\frac{1}{r^{2}}-\frac{1}{a_{I} a_{J}}-\frac{1}{a_{I} a_{K}}\right) \frac{r}{r_{J} r_{K}} d\left(\frac{1}{r_{I}}\right) \\
& +\left(-\frac{1}{r^{2}}+\frac{1}{a_{I} a_{J}}+\frac{1}{a_{I} a_{K}}+\frac{1}{a_{J} a_{K}}\right) \frac{r^{2}}{r_{I} r_{K} r_{K}} d\left(\frac{1}{r}\right) .
\end{aligned}
$$


We find

$$
\begin{aligned}
T^{(0)} & \equiv \cos \theta d \phi, \quad T_{I}^{(1)} \equiv \cos \theta_{I} d \phi, \quad T_{I}^{(2)} \equiv \frac{r-a_{I} \cos \theta}{r_{I}} d \phi, \\
T_{I J}^{(3)} & \equiv \frac{a_{I}}{a_{J}-a_{I}} \frac{r^{2}+a_{I} a_{J}-\left(a_{I}+a_{J}\right) r \cos \theta}{r_{I} r_{J}} d \phi, \\
T_{I J}^{(4)} & \equiv \frac{\left(r^{2}+a_{I} a_{J}\right) \cos \theta-\left(a_{I}+a_{J}\right) r}{r_{I} r_{J}} d \phi, \\
T_{I J K}^{(5)} & \equiv \frac{r^{3}+r\left(a_{I} a_{J}+a_{I} a_{K}+a_{J} a_{K}\right)-\left(r^{2}\left(a_{I}+a_{J}+a_{K}\right)+a_{I} a_{J} a_{K}\right) \cos \theta}{r_{I} r_{J} r_{K}} d \phi .
\end{aligned}
$$

Thus, $v_{0}$ and $v_{I}$ are given by

$$
\begin{aligned}
& v_{I}=\frac{\left|\epsilon_{I J K}\right|}{2}[-q k_{\infty}^{J} k_{\infty}^{K} T^{(0)}+2 k_{\infty}^{J} \kappa^{K} T_{J}^{(1)}-2 Q_{J}^{(I)} T_{J}^{(2)} \\
&\left.-\frac{\kappa_{J} \kappa_{K}}{q} T_{J K}^{(3)}\right], \\
& v_{0}=\frac{\left|\epsilon_{I J K}\right|}{6}\left[q k_{\infty}^{I} k_{\infty}^{J} k_{\infty}^{K} T^{(0)}+6 k_{\infty}^{I} Q_{J}^{(I)} T_{J}^{(1)}-3 k_{\infty}^{I} k_{\infty}^{J} \kappa_{K} T_{K}^{(2)}\right. \\
&\left.+6 \frac{\kappa_{I} Q_{J}^{(I)}}{q} T_{I J}^{(3)}+3 \frac{k_{\infty}^{I} \kappa_{J} \kappa_{K}}{q} T_{J K}^{(4)}+\frac{\kappa_{I} \kappa_{J} \kappa_{K}}{q^{2}} T_{I J K}^{(5)}\right] .
\end{aligned}
$$

\section{B The explicit asymptotically $\mathrm{WAdS}_{3} \times \mathrm{SqS}^{3}$ bubbling solution}

In this section, we focus on the IR geometry of the solution constructed in section 4.4. We give the local $\mathrm{S}^{1} \times \mathbb{R}^{4}$ metric (4.15) at each of the four centers and the periodicities of the angles.

- At the origin of the space, $r \sim 0$ :

The local metric is

$$
\begin{aligned}
d s_{0}^{2}=\frac{5 \Lambda^{2}}{18} \sqrt{\frac{7}{3}} & {\left[\frac{d r^{2}}{r}+\frac{5632}{1575} d y_{0}^{2}\right.} \\
& \left.+r\left(d \theta^{2}+2(1+\cos \theta) d \phi_{L 0}^{2}+2(1-\cos \theta) d \phi_{R 0}^{2}\right)\right],
\end{aligned}
$$

where $y_{0}, \phi_{L 0}$ and $\phi_{R 0}$ are related to the angles at infinity $y_{\infty}, \psi_{\infty}$ and $\phi$ by

$$
\begin{aligned}
y_{0} & =y_{\infty}+\frac{9}{4}\left(\psi_{\infty}-\phi\right), \\
\phi_{L 0} & =\frac{1}{8}\left(21 y_{\infty}+31\left(\psi_{\infty}-\phi\right)\right)+\phi, \\
\phi_{R 0} & =\frac{1}{8}\left(21 y_{\infty}+31\left(\psi_{\infty}-\phi\right)\right) .
\end{aligned}
$$


We can read the periodicities from (4.23)

$$
\left(y_{0}, \phi_{L 0}, \phi_{R 0}\right)=\left\{\begin{array}{l}
\left(y_{0}, \phi_{L 0}, \phi_{R 0}\right)-\frac{20 \pi T}{473}(51,64,64) \\
\left(y_{0}, \phi_{L 0}, \phi_{R 0}\right)+\frac{\pi}{2}(18,31,31) \\
\left(y_{0}, \phi_{L 0}, \phi_{R 0}\right)+2 \pi(0,1,0)
\end{array} .\right.
$$

Using the procedure detailed in section 4.2, this corresponds to a smooth discrete quotient of $\mathrm{S}^{1} \times \mathbb{R}^{4}$ if $b$ is not divisible by 2 where $b$ is the denominator of the irreducible fraction $T=\frac{a}{b}$.

- At the second center, $r_{1} \sim 0$ :

The local metric is

$$
\begin{aligned}
d s_{1}^{2}=\frac{\Lambda^{2}}{23} \sqrt{\frac{155}{2}} & {\left[\frac{d r_{1}^{2}}{r_{1}}+\frac{15548}{837} d y_{1}^{2}\right.} \\
& \left.+r_{1}\left(d \theta_{1}^{2}+2\left(1+\cos \theta_{1}\right) d \phi_{L 1}^{2}+2\left(1-\cos \theta_{1}\right) d \phi_{R 1}^{2}\right)\right],
\end{aligned}
$$

where $y_{1}, \phi_{L 1}$ and $\phi_{R 1}$ are related to the angles at infinity $y_{\infty}, \psi_{\infty}$ and $\phi$ by

$$
\begin{aligned}
y_{1} & =y_{\infty}+\psi_{\infty}-\frac{5}{13} \phi, \\
\phi_{L 1} & =\frac{1}{6}\left(21 y_{\infty}+31 \psi_{\infty}-23 \phi\right)+\phi, \\
\phi_{R 1} & =\frac{1}{6}\left(21 y_{\infty}+31 \psi_{\infty}-23 \phi\right) .
\end{aligned}
$$

We can read the periodicities from (4.23)

$$
\left(y_{1}, \phi_{L 1}, \phi_{R 1}\right)=\left\{\begin{array}{l}
\left(y_{1}, \phi_{L 1}, \phi_{R 1}\right)-\frac{160 \pi T}{1419}(3,32,32) \\
\left(y_{1}, \phi_{L 1}, \phi_{R 1}\right)+\frac{2 \pi}{3}(6,31,31) \\
\left(y_{1}, \phi_{L 1}, \phi_{R 1}\right)+\frac{2 \pi}{39}(24,91,52)
\end{array} .\right.
$$

This corresponds to a smooth discrete quotient of $\mathrm{S}^{1} \times \mathbb{R}^{4}$ if $b$ is not divisible by 13 where $b$ is the denominator of the irreducible fraction $T=\frac{a}{b}$.

- At the third center, $r_{5} \sim 0$ :

The local metric is

$$
\begin{aligned}
d s_{5}^{2}=\frac{65 \Lambda^{2}}{207} \sqrt{\frac{11}{6}} & {\left[\frac{d r_{5}^{2}}{r_{5}}+\frac{599081}{128700} d y_{5}^{2}\right.} \\
& \left.+r_{5}\left(d \theta_{5}^{2}+2\left(1+\cos \theta_{5}\right) d \phi_{L 5}^{2}+2\left(1-\cos \theta_{5}\right) d \phi_{R 5}^{2}\right)\right],
\end{aligned}
$$


where $y_{5}, \phi_{L 5}$ and $\phi_{R 5}$ are related to the angles at infinity $y_{\infty}, \psi_{\infty}$ and $\phi$ by

$$
\begin{aligned}
y_{5} & =y_{\infty}-\frac{3}{61}\left(3 \psi_{\infty}-13 \phi\right), \\
\phi_{L 5} & =\frac{1}{32}\left(21 y_{\infty}+31 \psi_{\infty}-17 \phi\right)+\phi, \\
\phi_{R 5} & =\frac{1}{32}\left(21 y_{\infty}+31 \psi_{\infty}-17 \phi\right) .
\end{aligned}
$$

Then, we can read the periodicities from (4.23)

$$
\left(y_{5}, \phi_{L 5}, \phi_{R 5}\right)=\left\{\begin{array}{l}
\left(y_{5}, \phi_{L 5}, \phi_{R 5}\right)+\frac{320 \pi T}{28853}(120,-61,-61) \\
\left(y_{5}, \phi_{L 5}, \phi_{R 5}\right)+\frac{\pi}{488}(-288,1891,1891) \\
\left(y_{5}, \phi_{L 5}, \phi_{R 5}\right)+\frac{\pi}{488}(480,1403,427)
\end{array} .\right.
$$

This corresponds to a smooth discrete quotient of $\mathrm{S}^{1} \times \mathbb{R}^{4}$ if $b$ is not divisible by 16 where $b$ is the denominator of the irreducible fraction $T=\frac{a}{b}$.

- At the fourth center, $r_{p} \sim 0$ :

The local metric is

$$
\begin{aligned}
d s_{p}^{2}=\frac{\Lambda^{2}}{207} \sqrt{\frac{145}{3}} & {\left[\frac{d r_{p}^{2}}{r_{p}}+\frac{66309}{232} d y_{p}^{2}\right.} \\
& \left.+r_{p}\left(d \theta_{p}^{2}+2\left(1+\cos \theta_{p}\right) d \phi_{L p}^{2}+2\left(1-\cos \theta_{p}\right) d \phi_{R p}^{2}\right)\right],
\end{aligned}
$$

where $y_{p}, \phi_{L p}$ and $\phi_{R p}$ are related to the angles at infinity $y_{\infty}, \psi_{\infty}$ and $\phi$ by

$$
\begin{aligned}
y_{p} & =y_{\infty}-\frac{21}{31}\left(\psi_{\infty}+\phi\right), \\
\phi_{L p} & =\frac{1}{16}\left(21 y_{\infty}+31 \psi_{\infty}+15 \phi\right)+\phi, \\
\phi_{R p} & =\frac{1}{16}\left(21 y_{\infty}+31 \psi_{\infty}+15 \phi\right) .
\end{aligned}
$$

Then, we can read the periodicities from (4.23)

$$
\left(y_{p}, \phi_{L p}, \phi_{R p}\right)=\left\{\begin{array}{l}
\left(y_{p}, \phi_{L p}, \phi_{R p}\right)+\frac{640 \pi T}{14663}(3,-31,-31) \\
\left(y_{p}, \phi_{L p}, \phi_{R p}\right)+\frac{\pi}{124}(336,961,961) \\
\left(y_{p}, \phi_{L p}, \phi_{R p}\right)+\frac{\pi}{124}(336,961,713)
\end{array} .\right.
$$

This corresponds to a smooth discrete quotient of $\mathrm{S}^{1} \times \mathbb{R}^{4}$ if $b$ is not divisible by 8 where $b$ is the denominator of the irreducible fraction $T=\frac{a}{b}$.

Consequently, the IR bubbling geometry is smooth if and only if $T=\frac{a}{b} \in \mathbb{Q}$ and $b$ is not divisible by 2 or 13 . 


\section{The explicit asymptotically NHEK bubbling solution}

In this section, we focus on the IR geometry of the solution constructed in 4.4. We give the local $S^{1} \times \mathbb{R}^{4}$ metrics (4.15) around each of the four centers and the periodicities of the angles.

- At the origin of the space, $r \sim 0$ :

The local metric is

$$
\begin{aligned}
d s_{0}^{2}=\frac{\Lambda^{2}}{240} \sqrt{\frac{675-67 \sqrt{65}}{6}} & {\left[\frac{d r^{2}}{r}+\frac{49(2915+259 \sqrt{65})}{10240} d y_{0}^{2}\right.} \\
& \left.+r\left(d \theta^{2}+2(1+\cos \theta) d \phi_{L 0}^{2}+2(1-\cos \theta) d \phi_{R 0}^{2}\right)\right],
\end{aligned}
$$

where $y_{0}, \phi_{L 0}$ and $\phi_{R 0}$ are related to the angles at infinity $y_{\infty}, \psi_{\infty}$ and $\phi$ by

$$
\begin{aligned}
y_{0} & =y_{\infty}+\frac{17}{7}\left(\psi_{\infty}-\phi\right), \\
\phi_{L 0} & =-6 y_{\infty}+10\left(\phi-\psi_{\infty}\right)+\phi, \\
\phi_{R 0} & =-6 y_{\infty}+10\left(\phi-\psi_{\infty}\right) .
\end{aligned}
$$

The periodicities derived from (5.8) are

$$
\left(y_{0}, \phi_{L 0}, \phi_{R 0}\right)=\left\{\begin{array}{l}
\left(y_{0}, \phi_{L 0}, \phi_{R 0}\right)+2 \pi\left(-\frac{33}{7 \Lambda}, \frac{15}{\Lambda}, \frac{15}{\Lambda},\right) \\
\left(y_{0}, \phi_{L 0}, \phi_{R 0}\right)+2 \pi\left(\frac{34}{7},-20,-20\right) \\
\left(y_{0}, \phi_{L 0}, \phi_{R 0}\right)+2 \pi(0,1,0)
\end{array} .\right.
$$

Using the procedure in section 4.2 , this is a smooth discrete quotient of $\mathrm{S}^{1} \times \mathbb{R}^{4}$ for any rational $\Lambda$.

- At the second center, $r_{1} \sim 0$ :

The local metric is

$$
\begin{aligned}
d s_{1}^{2}=\frac{\Lambda^{2}}{420} \sqrt{\frac{-635+123 \sqrt{65}}{2}} & {\left[\frac{d r_{1}^{2}}{r_{1}}+\frac{289(2575+303 \sqrt{65})}{47360} d y_{1}^{2}\right.} \\
& \left.+r_{1}\left(d \theta_{1}^{2}+2\left(1+\cos \theta_{1}\right) d \phi_{L 1}^{2}+2\left(1-\cos \theta_{1}\right) d \phi_{R 1}^{2}\right)\right]
\end{aligned}
$$

where $y_{1}, \phi_{L 1}$ and $\phi_{R 1}$ are related to the angles at infinity $y_{\infty}, \psi_{\infty}$ and $\phi$ by

$$
\begin{aligned}
y_{1} & =y_{\infty}+\frac{1}{17}\left(23 \psi_{\infty}-27 \phi\right), \\
\phi_{L 1} & =-6 y_{\infty}-10 \psi_{\infty}+12 \phi, \\
\phi_{R 1} & =-6 y_{\infty}-10 \psi_{\infty}+11 \phi .
\end{aligned}
$$


The periodicities are

$$
\left(y_{1}, \phi_{L 1}, \phi_{R 1}\right)=\left\{\begin{array}{l}
\left(y_{1}, \phi_{L 1}, \phi_{R 1}\right)-2 \pi\left(-\frac{27}{17 \Lambda}, \frac{15}{\Lambda}, \frac{15}{\Lambda},\right) \\
\left(y_{1}, \phi_{L 1}, \phi_{R 1}\right)+2 \pi\left(\frac{46}{17},-20,-20\right) \\
\left(y_{1}, \phi_{L 1}, \phi_{R 1}\right)+2 \pi\left(-\frac{4}{17}, 2,1\right)
\end{array} .\right.
$$

This is a smooth discrete quotient of $\mathrm{S}^{1} \times \mathbb{R}^{4}$.

- At the third center, $r_{5} \sim 0$ :

The local metric is

$$
\begin{aligned}
d s_{5}^{2}=\frac{\Lambda^{2}}{560} \sqrt{\frac{9635-323 \sqrt{65}}{6}} & \frac{d r_{5}^{2}}{r_{5}}+\frac{529(1215+223 \sqrt{65})}{564480} d y_{5}^{2} \\
& \left.+r_{5}\left(d \theta_{5}^{2}+2\left(1+\cos \theta_{5}\right) d \phi_{L 5}^{2}+2\left(1-\cos \theta_{5}\right) d \phi_{R 5}^{2}\right)\right]
\end{aligned}
$$

where $y_{5}, \phi_{L 5}$ and $\phi_{R 5}$ are related to the angles at infinity $y_{\infty}, \psi_{\infty}$ and $\phi$ by

$$
\begin{aligned}
y_{5} & =y_{\infty}+\frac{1}{23}\left(\psi_{\infty}+3 \phi\right), \\
\phi_{L 5} & =-\frac{1}{21}\left(6 y_{\infty}+10 \psi_{\infty}+9 \phi\right)+\phi, \\
\phi_{R 5} & =-\frac{1}{21}\left(6 y_{\infty}+10 \psi_{\infty}+9 \phi\right),
\end{aligned}
$$

with the following periodicities

$$
\left(y_{5}, \phi_{L 5}, \phi_{R 5}\right)=\left\{\begin{array}{l}
\left(y_{5}, \phi_{L 5}, \phi_{R 5}\right)+2 \pi\left(\frac{51}{23 \Lambda}, \frac{5}{7 \Lambda}, \frac{5}{7 \Lambda}\right) \\
\left(y_{5}, \phi_{L 5}, \phi_{R 5}\right)+2 \pi\left(\frac{2}{23},-\frac{20}{21},-\frac{20}{21}\right) \\
\left(y_{5}, \phi_{L 5}, \phi_{R 5}\right)+2 \pi\left(\frac{4}{23}, \frac{2}{21},-\frac{19}{21}\right)
\end{array} .\right.
$$

This corresponds to a smooth discrete quotient of $\mathrm{S}^{1} \times \mathbb{R}^{4}$.

- At the fourth center, $r_{p} \sim 0$ :

The local metric is

$$
\begin{aligned}
d s_{p}^{2}=\frac{4 \Lambda^{2}}{9} \frac{1}{43675+5435 \sqrt{65}} & {\left[\frac{d r_{p}^{2}}{r_{p}}+\frac{45(6145+737 \sqrt{65})}{8} d y_{p}^{2}\right.} \\
& \left.+r_{p}\left(d \theta_{p}^{2}+2\left(1+\cos \theta_{p}\right) d \phi_{L p}^{2}+2\left(1-\cos \theta_{p}\right) d \phi_{R p}^{2}\right)\right]
\end{aligned}
$$


where $y_{p}, \phi_{L p}$ and $\phi_{R p}$ are related to the angles at infinity $y_{\infty}, \psi_{\infty}$ and $\phi$ by

$$
\begin{aligned}
y_{p} & =y_{\infty}+\frac{3}{5}\left(\psi_{\infty}+\phi\right), \\
\phi_{L p} & =-6 y_{\infty}-10 \psi_{\infty}-9 \phi, \\
\phi_{R p} & =-6 y_{\infty}-10 \psi_{\infty}-10 \phi .
\end{aligned}
$$

The periodicities are

$$
\left(y_{p}, \phi_{L p}, \phi_{R p}\right)=\left\{\begin{array}{l}
\left(y_{p}, \phi_{L p}, \phi_{R p}\right)-2 \pi\left(\frac{3}{5 \Lambda}, \frac{15}{\Lambda}, \frac{15}{\Lambda},\right) \\
\left(y_{p}, \phi_{L p}, \phi_{R p}\right)+2 \pi\left(\frac{6}{5},-20,-20\right) \\
\left(y_{p}, \phi_{L p}, \phi_{R p}\right)+2 \pi\left(\frac{6}{5},-19,-20\right)
\end{array} .\right.
$$

This corresponds to a smooth discrete quotient of $S^{1} \times \mathbb{R}^{4}$.

Consequently, the IR bubbling geometry is smooth for any rational values of $\Lambda$.

Open Access. This article is distributed under the terms of the Creative Commons Attribution License (CC-BY 4.0), which permits any use, distribution and reproduction in any medium, provided the original author(s) and source are credited.

\section{References}

[1] S.D. Mathur, The fuzzball proposal for black holes: an elementary review, Fortsch. Phys. 53 (2005) 793 [hep-th/0502050] [INSPIRE].

[2] I. Bena and N.P. Warner, One ring to rule them all... And in the darkness bind them?, Adv. Theor. Math. Phys. 9 (2005) 667 [hep-th/0408106] [InSPIRE].

[3] P. Berglund, E.G. Gimon and T.S. Levi, Supergravity microstates for BPS black holes and black rings, JHEP 06 (2006) 007 [hep-th/0505167] [INSPIRE].

[4] I. Bena and N.P. Warner, Black holes, black rings and their microstates, Lect. Notes Phys. 755 (2008) 1 [hep-th/0701216] [INSPIRE].

[5] I. Bena, N. Bobev, S. Giusto, C. Ruef and N.P. Warner, An infinite-dimensional family of black-hole microstate geometries, JHEP 03 (2011) 022 [Erratum ibid. 04 (2011) 059] [arXiv: 1006.3497] [INSPIRE].

[6] I. Bena, S. Giusto, R. Russo, M. Shigemori and N.P. Warner, Habemus superstratum! A constructive proof of the existence of superstrata, JHEP 05 (2015) 110 [arXiv:1503.01463] [INSPIRE].

[7] F. Denef and G.W. Moore, Split states, entropy enigmas, holes and halos, JHEP 11 (2011) 129 [hep-th/0702146] [INSPIRE].

[8] A. Sen, Walls of marginal stability and dyon spectrum in $N=4$ supersymmetric string theories, JHEP 05 (2007) 039 [hep-th/0702141] [INSPIRE].

[9] I. Bena, P. Heidmann and D. Turton, AdS $S_{2}$ holography: mind the cap, arXiv:1806.02834 [INSPIRE]. 
[10] J. de Boer, S. El-Showk, I. Messamah and D. Van den Bleeken, Quantizing $N=2$ multicenter solutions, JHEP 05 (2009) 002 [arXiv:0807.4556] [INSPIRE].

[11] G. Bossard and S. Katmadas, Floating JMaRT, JHEP 04 (2015) 067 [arXiv:1412.5217] [INSPIRE].

[12] I. Bena, G. Bossard, S. Katmadas and D. Turton, Non-BPS multi-bubble microstate geometries, JHEP 02 (2016) 073 [arXiv:1511.03669] [INSPIRE].

[13] I. Bena, G. Bossard, S. Katmadas and D. Turton, Bolting multicenter solutions, JHEP 01 (2017) 127 [arXiv: 1611.03500] [INSPIRE].

[14] G. Bossard, S. Katmadas and D. Turton, Two kissing bolts, JHEP 02 (2018) 008 [arXiv: 1711.04784] [INSPIRE].

[15] M. Guica, T. Hartman, W. Song and A. Strominger, The Kerr/CFT correspondence, Phys. Rev. D 80 (2009) 124008 [arXiv: 0809.4266] [INSPIRE].

[16] S. El-Showk and M. Guica, Kerr/CFT, dipole theories and nonrelativistic CFTs, JHEP 12 (2012) 009 [arXiv: 1108.6091] [INSPIRE].

[17] S. Detournay, T. Hartman and D.M. Hofman, Warped conformal field theory, Phys. Rev. D 86 (2012) 124018 [arXiv:1210.0539] [InSPIRE].

[18] J.C. Breckenridge, D.A. Lowe, R.C. Myers, A.W. Peet, A. Strominger and C. Vafa, Macroscopic and microscopic entropy of near extremal spinning black holes, Phys. Lett. B 381 (1996) 423 [hep-th/9603078] [INSPIRE].

[19] M. Cvetič and D. Youm, General rotating five-dimensional black holes of toroidally compactified heterotic string, Nucl. Phys. B 476 (1996) 118 [hep-th/9603100] [INSPIRE].

[20] M. Cvetič and F. Larsen, Near horizon geometry of rotating black holes in five-dimensions, Nucl. Phys. B 531 (1998) 239 [hep-th/9805097] [INSPIRE].

[21] I. Bena, M. Guica and W. Song, Un-twisting the NHEK with spectral flows, JHEP 03 (2013) 028 [arXiv: 1203.4227] [INSPIRE].

[22] I. Bena, N. Bobev and N.P. Warner, Spectral flow and the spectrum of multi-center solutions, Phys. Rev. D 77 (2008) 125025 [arXiv:0803.1203] [inSPIRE].

[23] I. Bena, L. Heurtier and A. Puhm, AdS $S_{3}$ : the NHEK generation, JHEP 05 (2016) 120 [arXiv: 1510.08055] [INSPIRE].

[24] K. Goldstein and S. Katmadas, Almost BPS black holes, JHEP 05 (2009) 058 [arXiv: 0812.4183] [INSPIRE].

[25] I. Bena, S. Giusto, C. Ruef and N.P. Warner, Multi-center non-BPS black holes: the solution, JHEP 11 (2009) 032 [arXiv:0908.2121] [INSPIRE].

[26] P. Heidmann, Four-center bubbled BPS solutions with a Gibbons-Hawking base, JHEP 10 (2017) 009 [arXiv:1703.10095] [INSPIRE].

[27] G. Dall'Agata, S. Giusto and C. Ruef, U-duality and non-BPS solutions, JHEP 02 (2011) 074 [arXiv: 1012.4803] [INSPIRE].

[28] J.E. McClintock, R. Shafee, R. Narayan, R.A. Remillard, S.W. Davis and L.-X. Li, The spin of the near-extreme Kerr black hole GRS $1915+105$, Astrophys. J. 652 (2006) 518 [astro-ph/0606076] [INSPIRE]. 
[29] O.J.C. Dias, R. Emparan and A. Maccarrone, Microscopic theory of black hole superradiance, Phys. Rev. D 77 (2008) 064018 [arXiv:0712.0791] [InSPIRE].

[30] M. Guica and A. Strominger, Microscopic realization of the Kerr/CFT correspondence, JHEP 02 (2011) 010 [arXiv: 1009.5039] [INSPIRE].

[31] W. Song and A. Strominger, D-brane construction of the 5D NHEK dual, JHEP 07 (2012) 176 [arXiv: 1105.0431] [INSPIRE].

[32] I. Bredberg, T. Hartman, W. Song and A. Strominger, Black hole superradiance from Kerr/CFT, JHEP 04 (2010) 019 [arXiv:0907.3477] [INSPIRE].

[33] D. Anninos, W. Li, M. Padi, W. Song and A. Strominger, Warped AdS 3 black holes, JHEP 03 (2009) 130 [arXiv: 0807.3040] [INSPIRE].

[34] D. Orlando and L.I. Uruchurtu, Warped anti-de Sitter spaces from brane intersections in type-II string theory, JHEP 06 (2010) 049 [arXiv: 1003.0712] [INSPIRE].

[35] W. Song and A. Strominger, Warped AdS 3 /dipole-CFT duality, JHEP 05 (2012) 120 [arXiv: 1109.0544] [INSPIRE].

[36] I. Bena, G. Dall'Agata, S. Giusto, C. Ruef and N.P. Warner, Non-BPS black rings and black holes in Taub-NUT, JHEP 06 (2009) 015 [arXiv:0902.4526] [INSPIRE].

[37] O. Vasilakis and N.P. Warner, Mind the gap: supersymmetry breaking in scaling, microstate geometries, JHEP 10 (2011) 006 [arXiv:1104.2641] [INSPIRE].

[38] G.W. Gibbons and N.P. Warner, Global structure of five-dimensional fuzzballs, Class. Quant. Grav. 31 (2014) 025016 [arXiv: 1305.0957] [INSPIRE].

[39] B.E. Niehoff and H.S. Reall, Evanescent ergosurfaces and ambipolar hyper-Kähler metrics, JHEP 04 (2016) 130 [arXiv: 1601.01898] [INSPIRE].

[40] J.P. Gauntlett and J.B. Gutowski, General concentric black rings, Phys. Rev. D 71 (2005) 045002 [hep-th/0408122] [INSPIRE].

[41] I. Bena, P. Kraus and N.P. Warner, Black rings in Taub-NUT, Phys. Rev. D 72 (2005) 084019 [hep-th/0504142] [INSPIRE].

[42] B. Bates and F. Denef, Exact solutions for supersymmetric stationary black hole composites, JHEP 11 (2011) 127 [hep-th/0304094] [INSPIRE].

[43] I. Bena, S. Giusto, C. Ruef and N.P. Warner, Supergravity solutions from floating branes, JHEP 03 (2010) 047 [arXiv:0910.1860] [INSPIRE].

[44] D. Mateos and P.K. Townsend, Supertubes, Phys. Rev. Lett. 87 (2001) 011602 [hep-th/0103030] [INSPIRE].

[45] I. Bena, N. Bobev, C. Ruef and N.P. Warner, Supertubes in bubbling backgrounds: Born-Infeld meets supergravity, JHEP 07 (2009) 106 [arXiv: 0812.2942] [INSPIRE].

[46] S. Giusto, O. Lunin, S.D. Mathur and D. Turton, D1-D5-P microstates at the cap, JHEP 02 (2013) 050 [arXiv: 1211.0306] [INSPIRE].

[47] B. Chakrabarty, D. Turton and A. Virmani, Holographic description of non-supersymmetric orbifolded D1-D5-P solutions, JHEP 11 (2015) 063 [arXiv:1508.01231] [INSPIRE]. 